\title{
Acoustic techniques for concrete evaluation: Improvements, comparisons and consistency
}

\author{
Vincent Garnier ${ }^{\mathrm{a}, *}$, Bogdan Piwakowski ${ }^{\mathrm{b}}$, Odile Abraham ${ }^{\mathrm{c}}$, Géraldine Villain ${ }^{\mathrm{c}}$, Cédric Payan ${ }^{\mathrm{a}}$, \\ Jean François Chaix ${ }^{a}$ \\ ${ }^{a}$ Aix Marseille Université, LMA, Aix en Provence, France \\ ${ }^{\mathrm{b}}$ Ecole Centrale Lille, IEMN, France \\ ${ }^{\mathrm{c}}$ Université de Nantes-Angers-Le Mans, IFSTTAR, Nantes, France
}

In civil engineering, the testing of structures allows a large number of chemical, mechanical and micromacro structural parameters to be determined. Within the scope of ultrasonic Non-Destructive Testing (NDT), which is often used to evaluate concrete, many different techniques can be employed to generate, propagate, and receive the ultrasound waves, and to process the corresponding signals. The SENSO project, developed by nine partners, provided an opportunity to test a large number of principles related to the material's characteristics and properties. Concerning the acoustic techniques, the first approach was based on linear propagation with bulk waves and surface waves, or with backscattered waves. The second method involved nonlinear analysis using wave modulation. One aim of the SENSO project was to compare these different techniques, in order to select that having the most promising performance in the laboratory, before transferring the measurement techniques to the field. The objectives were to evaluate the porosity, degree of saturation, modulus of elasticity and compressive strength of concrete. In the present paper, the measurement techniques and measured parameters are presented, together with the improvements developed within the context of the SENSO project. The methodology used to classify and select the ultrasonic techniques is described. It takes into account the sensitivity of the method to changes in the material and associates measurement uncertainties. Finally, the most significant results to evaluate porosity, mechanical characteristics and degree of saturation of various concretes, are developed. The consistency of the results of the measurements is analysed. The advantages and drawbacks of each technique are discussed.

\section{Investigation of concrete at various scales}

The French project SENSO [1], which was run from 2005 until 2009, has overcome certain scientific problems, in particular: the creation of a database, command of measurement techniques, selection of sustainability indicators of the concrete, choice of ND

* Corresponding author. Address: Laboratoire de Mécanique et d'Acoustique, Aix Marseille Université, Thème LCND, IUT Aix Provence, Avenue Gaston Berger, 13625 Aix en Provence Cedex 1, France. Tel.: +33 442939037; fax: +33 442939070.

E-mail address: vincent.garnier@univ-amu.fr (V. Garnier). parameters adapted to diagnosis and data inversion. This was achieved through the development of a set of not only conventional, but also original techniques, capable of affecting the material and its structure at all scales, which were implemented as part of an experimental design. This project, based on the examination of specimens conditioned under controlled conditions of porosity and saturation, allowed material properties to be derived from four techniques (radar, ultrasound, electrical resistivity, and capacitance measurements). This approach is the result of a multi-technical concept, which was developed in laboratory, to be 
implemented thereafter on site. A new project, referred to as: "Analysis and Capitalization for the Diagnosis of Constructions", supported by the MEDDTL (French Ministry for the environment, sustainable development, transport and housing) and RGCU (Civil Engineering and Urban French Network) has been selected to study the transfer of knowledge and methodologies from the laboratory to the case of on-site assessments.

Among the above-described techniques, the propagation of ultrasonic waves is often used alone as a first-line approach. In the present study, our analysis was limited to comparisons based on the use of ultrasound measurements.

Ultrasounds are mechanical waves which generate compression and shear movements of the particles. It is common to use ultrasonic waves to evaluate the material's characteristics and the pathologies of concrete in civil engineering. Various standards $[2,3]$ and recommendations [4] provide details on test methods. They often consist in developing relations between non-destructive (ND) parameters (the results provided by the measurements) and the indicators (the information describing the concrete's state). The present study has been confined to the determination of four sustainability indicators, which are: porosity, degree of saturation, modulus of elasticity and compressive strength. It is important for the conclusions of the study to be structured with respect to the different ND parameters and their ability to provide coherent information on the indicators during the investigation of concrete structures. The study was thus committed to:

- Comparing the ND parameters in terms of their repeatability, sensitivity, and representativeness.

- Selecting the most suitable ND parameters and techniques.

- Establishing relationships between the ultrasonic measurements (ND parameters) and the four indicators.

It is known that ultrasonic waves have numerous interactions with the material and that the data derived from such observations is related to the behaviour of materials at different microstructure scales. The sensitivity of ultrasonic waves to spatial and temporal variations of the material is therefore specific to the scale at which the interactions take place. The responses, together with their range and uncertainties, must be studied at each scale. The concept of multi-scale interactions can be described, starting at the level of atomic bonds, and including that of the mesoscopic (microscopic and millimetric) structure [5,6], the aggregate dimension, and finally the concrete structure as a single component. The advantage of ultrasound waves is that they allow access to be gained to every level of these interactions, thereby providing a reliable means of characterising the material. The propagation of acoustic waves is described at these different levels [7], and the behaviour of the material is summarised in the form of a propagation model combining both linear and nonlinear contributions.

The concrete linear behaviour governed the equations of ultrasonic wave propagation used in civil engineering that can be modified by multiple scattering in the case of interactions with aggregates. This phenomenon generates a "coda" $[8,9]$. The relevant linear parameters are the velocity and attenuation of the ultrasonic waves [10-13].

The material's nonlinear behaviour is driven by atomic bond, contact, cohesion/decohesion and capillarity phenomena. These influences have been defined through the use of a phenomenological description $[14,15]$.

For the purposes of the present study, specific devices were developed, to solicit linear and nonlinear reactions of the material. The techniques used for linear characterisation were based on the analysis of transmission, resonance, surface and backscattered waves, allowing the following parameters to be determined: velocity $V$, attenuation $\alpha$, and resonant frequency $f_{\text {res. }}$. Frequency modulation was used for the nonlinear characterisation, leading to the dimensionless nonlinear parameter $\alpha$ nl.

A second advantage of ultrasonic testing is that it allows different volumes of concrete to be analysed. Investigations can be made either through the full volume of the specimen, in order to retrieve global information (transmission, resonance and frequency modulation), or just at its surface (using surface waves and/or backscattered waves), to evaluate the cover concrete. The small thickness of the cover concrete and its ability to transport aggressive agents plays a very important role in the protection of the reinforcing bars.

In this paper, each of the above-described techniques is presented, together with its objectives in terms of the evaluation of the material under investigation and the parameters which can be derived from the measurements. Next, the methods to select the most suitable ND parameters for the characterisation of the material are developed. Finally, the evolutions of the different ND parameters as a function of the indicators tested in the SENSO project are compared.

An evaluation of the potential of these various techniques for on-site applications is provided in the conclusions.

\section{Experimental context}

\subsection{Experimental plan}

The aim of the ANR-SENSO project $[16,4]$ was to evaluate the indicators by nondestructive techniques on slabs. It was possible to model the relationships of the ND parameters with the indicators: degree of saturation, Young's modulus, porosity, and compressive strength. The indicators were considered to be homogeneous, throughout the slab. For our study, the analysed data was limited to that generated by six batches of concrete, made with the same aggregates (round, siliceous, $D_{\max }=14 \mathrm{~mm}$ ), with water to cement $(\mathrm{W} / \mathrm{C})$ ratios varying from 0.3 to 0.8 . This allowed different concretes to be obtained, with varying porosities (from 12.5\% to $18.1 \%$ ), moduli of elasticity (from $21,300 \mathrm{MPa}$ to $35,500 \mathrm{MPa}$ ), and concrete strengths (from 20 to $72.9 \mathrm{MPa}$ ) (Table 1). The sensitivity of ultrasound propagation and other non-destructive techniques to the concrete's characteristics were studied.

Each batch was composed of eight $50 \times 25 \times 12 \mathrm{~cm}$ parallelepiped slabs. The concrete samples were tested in the laboratory under controlled conditions of saturation, ranging from dry to saturated (0-30-50-70-100\%). Wet coupling was imposed for this saturation control. The cores used for destructive testing were extracted from two specific slabs [16].

The five ultrasonic parameters developed in the context of this project were: pressure waves (PW), surface waves (SW), backscattered waves (BW), impact echo (IE) and nonlinear analysis (NLA).

The contributions of each partner are described in Table 2.

\subsection{Geometry of the specimens}

The geometry of the above-described specimens made it possible to work with one technique at several points $(1,3$ or 10$)$ on each specimen. That allowed to analyse the repeatability of the measurements and the influence of spatial variability. The dimensions of the slab also made it possible to use automated devices. The specimen's geometry influences mainly the resonance frequencies of the Impact Echo and Frequency Modulation techniques.

Surface wave propagation techniques remain independent of the specimen's geometry, on two conditions. Firstly, the measurements should be confined to the first component of the signal, corresponding to the coherent wave. The non-coherent wave is produced by echoes from the edges and faces of the specimen in addition to the scattering effects from the aggregates. It is called the "coda" and arrives

Table 1

Characteristics of the concretes tested in the SENSO project in saturated conditions.

\begin{tabular}{llllll}
\hline $\begin{array}{l}\text { Reference of } \\
\text { batch }\end{array}$ & $\begin{array}{l}\text { W/C } \\
(\%)\end{array}$ & $\begin{array}{l}\text { Porosity } \\
(\%)\end{array}$ & $\begin{array}{l}\text { Modulus } \\
(\mathrm{GPa})\end{array}$ & $\begin{array}{l}\text { Strength } \\
(\mathrm{MPa})\end{array}$ & $\begin{array}{l}\text { Density } \\
\left(\mathrm{kg} / \mathrm{m}^{3}\right)\end{array}$ \\
\hline G1 & 0.31 & 12.5 & 35.5 & 72.9 & 2441 \\
G2 & 0.47 & 14.3 & 28.4 & 43.3 & 2469 \\
G3 & 0.59 & 15.5 & 27.7 & 43.8 & 2457 \\
G3a & 0.57 & 16 & 27.9 & 40.5 & 2447 \\
G7 & 0.63 & 15.9 & 27.4 & 38.3 & 2455 \\
G8 & 0.9 & 18.1 & 21.3 & 20.2 & 2405 \\
\hline
\end{tabular}


Table 2

Techniques implemented in the SENSO project and partners concerned.

\begin{tabular}{lll}
\hline Technique & Code & Partner \\
\hline Pressure waves & PW & LMA Aix - EC Lille \\
Surface waves & SW & IFSTTAR Nantes - EC Lille \\
Backscattered waves & BW & LMA Aix \\
Impact Echo & IE & IFSTTAR Nantes \\
Nonlinear analysis & NLA & LMA Aix \\
\hline
\end{tabular}

later in the signal. It was not studied in this project. Secondly, the ultrasound wavelength $\lambda$ is chosen to be much smaller than the thickness of the slab, so that the wave does not propagate across its entire thickness. Otherwise, if the entire volume of the specimen is affected, Lamb waves are generated. Frequencies ranging from a few tens of $\mathrm{kHz}$ up to $250 \mathrm{kHz}$ were used in the tests. The depths of investigation varied from 10 to $50 \mathrm{~mm}$ for the surface waves, under the assumption of an average surface wave velocity of approximately $2500 \mathrm{~m} / \mathrm{s}$.

The backscattered waves remained independent of the specimen's dimensions, because the studied zone was small with a depth varying, as a function of frequency, between 30 and $100 \mathrm{~mm}$.

Most of the ultrasound techniques allowed measurements to be made at the three test points identified on each specimen.

\subsection{Frequency and attenuation}

The frequency $f$ of the wave is a very important measurement parameter. The size of the aggregates, $D_{\max }=a$ and the wavelength $\lambda=V / f$ are related to the wave number $k=2 \pi / \lambda$ and allow the attenuation to be quantified by the following equations:

$k a \ll 1 \quad$ Rayleigh field $\alpha(f)=b_{1} \cdot f+c_{1} \cdot a^{3} \cdot f^{4}$

$k a \approx 1$ Stochastic field $\alpha(f)=b_{2} \cdot f+c_{2} \cdot a^{1} \cdot f^{2}$

$k a \gg 1$ Geometric field $\alpha(f)=b_{3} \cdot f+c_{3} \cdot a^{-1} \cdot f^{0}$

The coefficients $b_{1}, b_{2}, b_{3}, c_{1}, c_{2}, c_{3}$ are specific to the material. $b_{1}, b_{2}, b_{3}$ are related to the absorption, which was assumed to be low in concrete for our measurements. $c_{1}, c_{2}, c_{3}$ are related to the diffusion induced by the aggregates.

For transmission or surface techniques, a variable frequency parameter ranging between 50 and $250 \mathrm{kHz}$, corresponding to wavelengths ranging between approximately 10 and $100 \mathrm{~mm}$, was used, depending on the type of wave. For the aggregates, whose size did not exceed $D_{\max }=14 \mathrm{~mm}$, ka varied from 1 to 10 . Thus, in most cases the tests were carried out in the stochastic field, such that the concrete behaved like a dispersive material. A consequence of this interaction is that the ultrasound group velocity, the phase velocity and the attenuation vary with frequency. A second consequence is the production of multi-scattered waves that generated backscattered waves and transmitted waves with a coda. In this study, only the backscattered component was used.

In addition, it is known that in order to affect increasingly small elements of the microstructure, one possible solution is to increase the frequency. As the attenuation is a quadratic function of frequency, it rapidly becomes too high to allow the waves to be transmitted over significant distances.

In conclusion, it is important to specify that concrete can be heterogeneous. At the scale of the slab, this can be observed in the form of segregation and a porosity or moisture content gradient as a function of depth. In several cases, this characteristic led the authors to study the concrete in terms of the phase velocity and attenuation of the transmitted waves.

\subsection{Investigated volume}

Pressure waves can be used to determine: the ultrasound velocity of transmitted waves, the resonant frequency excited by the Impact Echo, the attenuation (based on the measurement of backscattered waves), and also the nonlinear parameter.
The volume of concrete affected by the wave needs to be defined for each technique (Fig 1). The first arrival time is determined using the transmitted wave, which propagates in 3D. Only those components of the wave which traverse the slab via the shortest path, and are recorded by the receiver, contribute to this time determination. This allowed the volume affected by the wave to be considered as a cylinder shape. Its size is that of the transducer. Two techniques were used to make the slab resonate: the Impact Echo technique and, as explained in the following chapter, nonlinear measurements. The volume affected by resonance was considered to be that of the entire slab. Surface waves affect the specimen down to a depth similar to the wavelength $[17,18]$. Analysis of these waves allowed the velocity and attenuation to be determined as a function of frequency. The backscattered waves, produced by reflection and scattering of the wave by the aggregates, were found to affect the concrete down to a depth varying between 30 and $100 \mathrm{~mm}$. This information was used to determine the attenuation coefficient of each specimen.

The volume of the specimens is an important parameter for the test, since it can affect its accuracy, in particular when the ultrasonic beam is smaller than the mean size of the aggregates. In the experiments described here, the diameter of the transducers ranged between 10 and $50 \mathrm{~mm}$. In the case of pressure waves, the ultrasonic beam has a diameter two or three times the dimensions of the aggregates. The presence of the latter can thus affect the wave's propagation time. On a larger scale, a varying distribution of aggregates can therefore have a varying influence on the measurements. Since the aggregates are randomly distributed, it is necessary to determine an average value from several measurements, made at different points, in order to take the influence of independent aggregate distributions [13] into account, and evaluate the concrete's mean properties.

A second example is given by surface waves penetrating to a depth [19] in the range between 10 and $50 \mathrm{~mm}$, which corresponds to the approximate size of the aggregates. Several profiles were generated by moving the receiver transducer, to vary the statistical distribution of the aggregates resulting from these displacements. However, the best approach to recording independent observations is to change the device's position [20]. The number of profiles needed to determine a mean value then depends on the desired statistical accuracy of this result [21]. In addition, the concrete's near-surface properties can vary with depth, and a gradient in these properties can modify the wave's propagation.

To conclude, one difficulty encountered when comparing the various measurement techniques was that of processing information from non-identical volumes or areas. So, it was necessary to include statistical analyses in the study, in order to take the concrete's spatial variability into account.

\section{Techniques}

Five techniques and several set-ups were used, as briefly described in the following. From the experimental data, 23 ND parameters were derived and thoroughly studied. Table 3 provides a summary of these parameters, with theirs codes, descriptions, and the mean values obtained from each type of measurement. The variation coefficient (VC) and quality index (QI) are used to evaluate the quality of the ND measurements, as described in detail in Section 4. The last column corresponds to the $\mathrm{Q}$ classification.

\subsection{Pressure waves}

Propagation using bulk pressure wave transmission techniques is now used on a regular basis for concrete inspection in Civil Engineering. Our tests were performed by two different research partners, using the setup shown in Fig. 2.

One of these partners works with industrial equipment, using two $50 \mathrm{kHz}$ transducers with no coupling. This instrument mea-

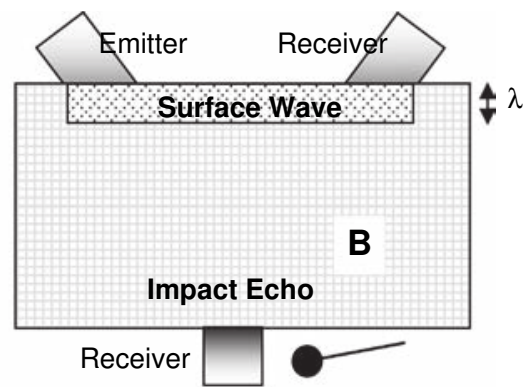

Fig. 1. Presentation of the different volumes investigated by the different types of waves on a concrete slab. 
Table 3

List of the Non-Destructive Parameters with the Code, Mean value of the ND parameter, Mean Variation Coefficient, Quality Index and CLassification (regarding the QI).

\begin{tabular}{|c|c|c|c|c|c|}
\hline Code & ND parameter & Mean NDp & Mean VC & QI & $\mathrm{CL}$ \\
\hline PW IE & Pressure Wave Velocity by impact echo $(\mathrm{m} / \mathrm{s})$ & 3972 & 2.85 & 2.75 & 1 \\
\hline IE $v$ & Dynamic Poisson's Ratio by Impact echo & 0.19 & 15.1 & 1.27 & 2 \\
\hline $\mathrm{SW} 4 \mathrm{~cm}$ & Surface Wave Velocity with $\lambda=4 \mathrm{~cm}(\mathrm{~m} / \mathrm{s})$ & 2211 & 1.83 & 1.21 & 3 \\
\hline$S W 3 \mathrm{~cm}$ & Surface Wave Velocity with $\lambda=3 \mathrm{~cm}(\mathrm{~m} / \mathrm{s})$ & 2214 & 1.79 & 1.17 & 4 \\
\hline PW 250 & Pressure Wave Velocity $(\mathrm{m} / \mathrm{s})$ by Transmission at $250 \mathrm{kHz}$ & 4413 & 1.62 & 1.08 & 5 \\
\hline IE Edyn & Dynamic Elastic Modulus by Impact echo (GPa) & 34.7 & 3.68 & 1.05 & 6 \\
\hline $\mathrm{SW} 2 \mathrm{~cm}$ & Surface Wave Velocity with $\lambda=2 \mathrm{~cm}(\mathrm{~m} / \mathrm{s})$ & 2221 & 2.00 & 0.88 & 7 \\
\hline $\mathrm{SW} 5 \mathrm{~cm}$ & Surface Wave Velocity with $\lambda=5 \mathrm{~cm}(\mathrm{~m} / \mathrm{s})$ & 2207 & 1.81 & 0.88 & 8 \\
\hline $\mathrm{SW} 1 \mathrm{~cm}$ & Surface Wave Velocity with $\lambda=1 \mathrm{~cm}(\mathrm{~m} / \mathrm{s})$ & 2232 & 2.56 & 0.78 & 9 \\
\hline SW at & Surface Wave Attenuation (dB/m) & 134 & 23.1 & 0.70 & 10 \\
\hline SW sd-at & Standard Deviation of Surface Wave Attenuation $(\mathrm{dB} / \mathrm{m})$ & 17.1 & 29.8 & 0.68 & 11 \\
\hline BW 2M & Backscattered Wave attenuation for $2 \mathrm{MHz}(\mathrm{Np} / \mathrm{m})$ & 15.8 & 21.1 & 0.68 & 12 \\
\hline SW qf & Quality Factor of Surface Wave & 19.7 & 29.4 & 0.59 & 13 \\
\hline$S W$ qf $2 \mathrm{~cm}$ & Quality Factor of Surface Wave with $\lambda=2 \mathrm{~cm}$ & 32.9 & 39.3 & 0.53 & 14 \\
\hline SW sd me & Standard Deviation Mean Velocity of Surface Wave $(\mathrm{m} / \mathrm{s})$. & 57.6 & 57.7 & 0.47 & 15 \\
\hline NLA & Nonlinear Acoustic coefficient (modulation of frequency) & 86.7 & 39.1 & 0.45 & 16 \\
\hline BW $1 \mathrm{M}$ & Backscattered Wave attenuation for $1 \mathrm{MHz}(\mathrm{Np} / \mathrm{m})$ & 16.3 & 14.8 & 0.44 & 17 \\
\hline$S W$ qf $1 \mathrm{~cm}$ & Quality Factor of Surface Wave with $\lambda=1 \mathrm{~cm}$ & 22.9 & 16.6 & 0.40 & 18 \\
\hline SW me & Mean Velocity of Surface Wave $(\mathrm{m} / \mathrm{s})$ & 2071 & 3.1 & 0.24 & 19 \\
\hline SW ap & Apparent Surface Wave Velocity $(\mathrm{m} / \mathrm{s})$. & 2220 & 2.29 & -10.0 & 20 \\
\hline PW 50 & Pressure Wave Velocity $(\mathrm{m} / \mathrm{s})$ by Transmission at $50 \mathrm{kHz}$ & 4129 & 2.98 & -10.0 & 21 \\
\hline PW sd 50 & Standard Deviation of Pressure Wave PW $50 \mathrm{kHz}$ & 1.95 & 43.7 & -10.0 & 22 \\
\hline SW qf ap & Quality Factor of Apparent Surface Wave & 21.5 & 33.2 & -10.0 & 23 \\
\hline
\end{tabular}

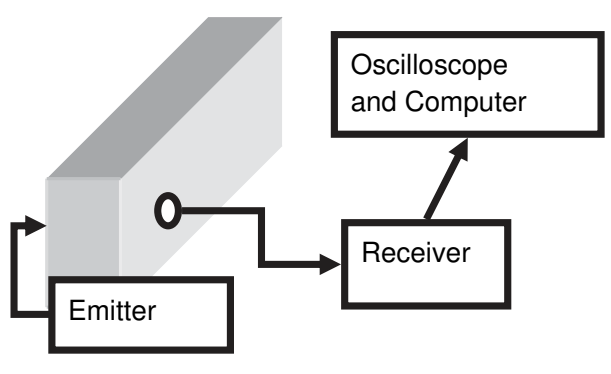

Fig. 2. Transmission principle.

sures travel times, $\Delta t$, based on measurements made at two different locations (longitudinal and transverse) in the specimen.

$V=\frac{L}{\Delta t}$.

The ND parameters are the Pressure Wave velocity : PW $50(\mathrm{~m} /$ $\mathrm{s}$ ) and the standard deviation of the Pressure Wave velocity : PW sd $50(\mathrm{~m} / \mathrm{s})$.

A second SENSO partner works with transducers having a central frequency of $250 \mathrm{kHz}$. The wave is transmitted across the $12 \mathrm{~cm}$ thickness of the slab, and in this case the coupling is achieved through silicone grease applied to adhesive tape, which is stuck onto the surface to protect the concrete. For the tests at $250 \mathrm{kHz}$, the averaging over random aggregate distributions was based on the acquisition of 100 signals, recorded by displacing the transducer around the perimeter of a circle twice as large as the size of the transducer. The group velocity was determined by extracting the time of flight, $\Delta t$, from the averaged signal.

The ND parameter is the Pressure Wave Velocity at $250 \mathrm{kHz}$ : PW $250(\mathrm{~m} / \mathrm{s})$.

\subsection{Backscattered waves}

These are generated and received by means of a single transducer or dual piezoelectric patches (one transmitter and one receiver). The signal produced by summing all the backscattered contributions is shown in Fig. 3.

Backscattered wave tests are in principle straightforward to implement, because they require only one lateral access point and one transducer. The backscattered signal $g(t)$ received by the transducer following transmission, reflection and scattering can be defined [22] by the function:

$r(t)=u_{0} \cdot e^{-\alpha \cdot \cdot V \cdot t} \cdot \sum_{k=1}^{N} \sigma_{k} \cdot e^{i \cdot \varphi_{k}}$

where $u_{0}$ is the initial amplitude of the wave, $\alpha r$ is the wave attenuation coefficient, $V$ is the velocity of ultrasonic compression waves in concrete, $t$ is the time, $N$ is the number of scatterers (the aggregates), $\sigma_{k}$ is the diffusion cross-section, which depends on the characteristics of the diffuser $k$, and $\varphi_{k}=\omega \cdot\left(t-\tau_{k}\right)$ is the temporal position of the scatterer $k$ Since the signal is in the far field zone and the aggregates are randomly distributed, it is possible to approximate the exponential decay curve $-\alpha r \cdot V \cdot t$ by an envelope (Fig. 3). Knowledge of the previously determined velocity $V$ allows
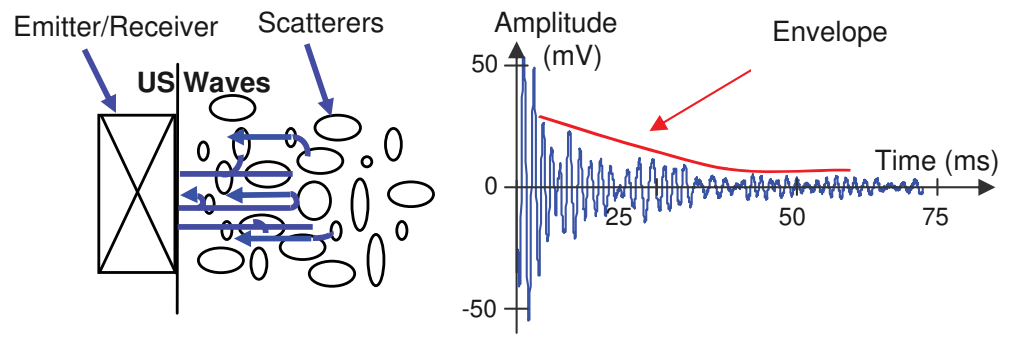

Fig. 3. Backscattered wave principle. 

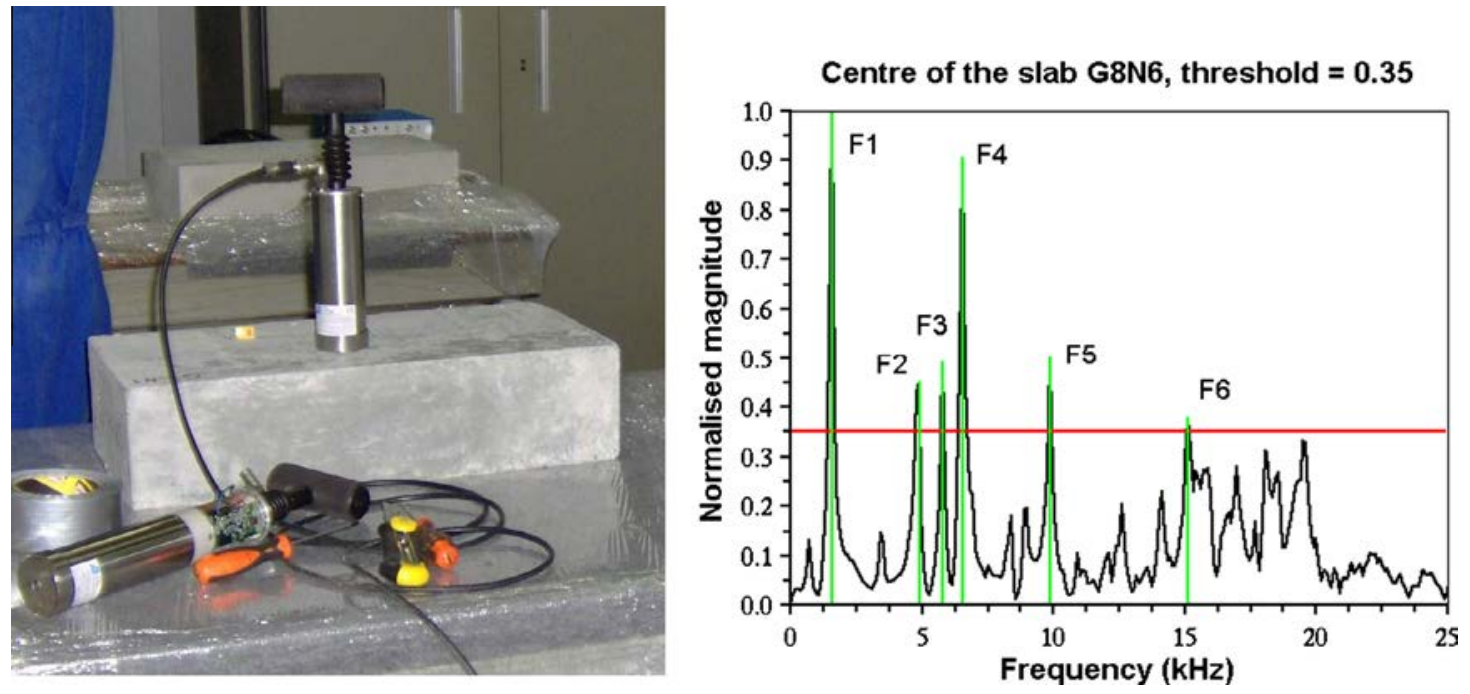

Fig. 4. Impact Echo principle and spectrum obtained from a concrete slab.

the attenuation coefficient of the backscattered wave to be estimated [23]. The tests were conducted at 1 and $2 \mathrm{MHz}$, which is higher than the usual frequencies specified above.

The ND parameters are respectively: BW $1 \mathrm{M}(\mathrm{Np} / \mathrm{m})$ and BW $2 \mathrm{M}(\mathrm{Np} / \mathrm{m})$.

\subsection{Impact Echo}

The Impact Echo technique was developed to detect voids in building materials and to measure the thickness of slabs [24]. A mechanical impact generates mechanical vibrations in a concrete structure. The resonant frequencies are measured by an ultrasonic transducer or an accelerometer. For a slab assumed to have infinite dimensions, the detected frequency corresponds to a pseudo-stationary symmetric mode (S1) of the guided Lamb waves [25]:

$f_{S 1}=\beta V / 2 e$

where $V$ is the compression wave velocity $(\mathrm{m} / \mathrm{s})$ and $e$ is the thickness of the plate $(\mathrm{m})$. The coefficient $\beta$ was initially estimated using an empirical technique described by Sansalone and Streett [25], who considered it as a shape factor. Gibson and Popovics [26] later showed that the coefficient $\beta$ can be calculated as a function of the Poisson's ratio $v$ of the material.

The impact is generated by a steel ball, thus in the absence of coupling (Fig. 4), and the measurement is made at approximately $20 \mathrm{~mm}$ from the point of impact. The information extracted from the spectrum derived from each slab, and from each type of concrete, is identical: six frequencies (denoted Fi in Fig. 4) are identified. In view of the rectangular geometry of the specimens, the specific resonant frequencies of the slabs should be studied. From the six resonant frequencies $\mathrm{Fi}$, it is possible to associate the frequencies F1, F2, F3 with the beam's first three resonance modes, calculated using the model developed by Timoshenko [27]. In addition, although the infinite slab assumption is no longer valid, [27] have shown that it is also possible to observe the first symmetric mode of Lamb waves in slabs with these dimensions, and to associate this S1 mode with the measured frequency F6. As a consequence of the stationarity of this mode, [28] has explained that it is weakly influenced by edge effects.

Thus, the theoretical frequencies (denoted fi) depend only on the dynamic Young's modulus, the Poisson's ratio and the known slab dimensions. By minimising the difference between the theoretical and experimental frequencies $|\mathrm{Fi}-\mathrm{fi}|$ (for $\mathrm{i}=1,2,3,6$ ), and

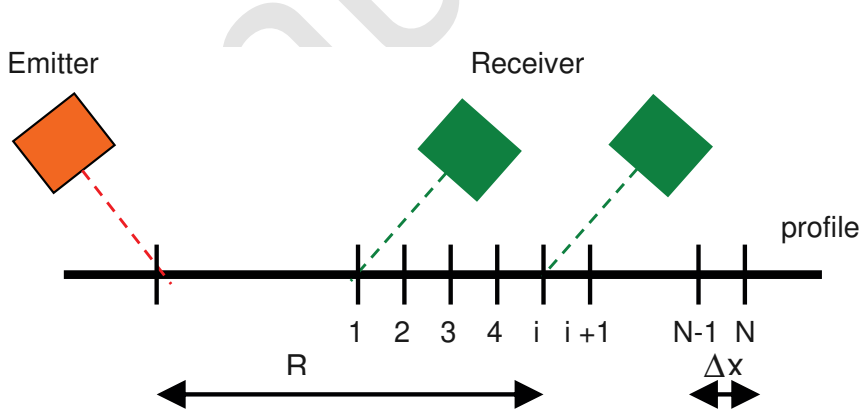

Fig. 5. Surface wave measurement principle.

ensuring that the difference $\mid \mathrm{Fi}$ - fi| remained below $50 \mathrm{~Hz}$ (frequency sampling), it was possible to evaluate the dynamic modulus of elasticity Edyn and Poisson's ratio $v$, following which the velocities of the compression and shear waves, and their uncertainties, could be determined. For the nine types of concrete, the studied frequencies ranged between 1.5 and $19 \mathrm{kHz}$. The ND parameters are the dynamic modulus of elasticity: IE Edyn (GPa), the dynamic Poisson's ratio: IE $v$, and the Pressure Wave velocity obtained from the Impact Echo test: PW IE (m/s).

\subsection{Surface waves}

The principle of the surface wave measurements is shown in Fig. 5. Two transducers are oriented at the critical angle [29], one of which can be translated along a line (profile) to different positions $R_{i}$. For a single transducer, the displacement $\Delta x$ and the time difference $\Delta t$ between the points allow the velocity and attenuation of the wave to be calculated. Concrete is a dispersive material, such that the phase velocity $V_{p h}(\omega)$ and group velocity $V_{g r}(\omega)$ are frequency dependent.

The surface waves referred to as Rayleigh waves, have three advantages:

- they allow one side of the structure to be accessed,

- they are highly sensitive to changes in the concrete's mechanical properties,

- they are characterised by a depth of penetration equivalent to the wavelength. Variations in the concrete's properties (moisture content, porosity, modulus of elasticity) as a function of 


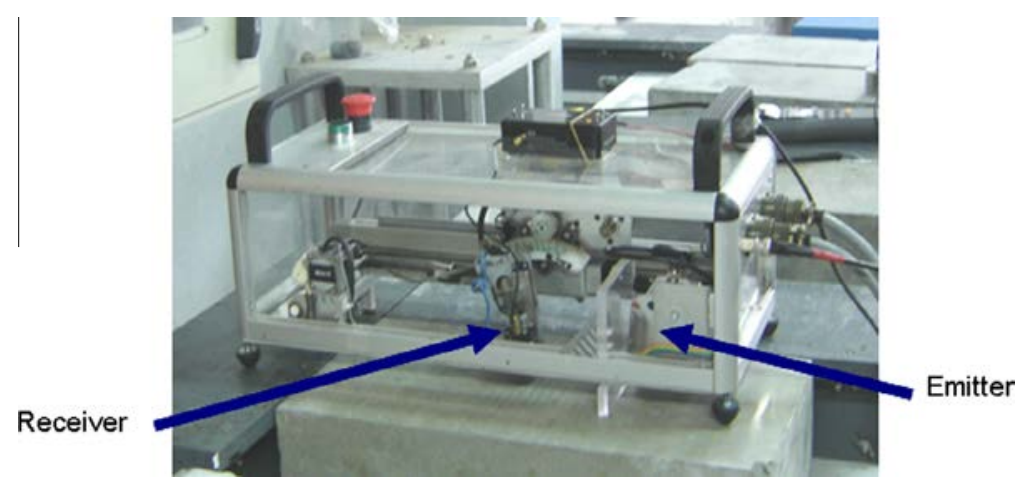

Fig. 6. Robot to generate and receive the surface waves.

depth can indeed be studied with the corresponding frequencies. In the context of the SENSO project, the ND parameters were defined for chosen frequencies or wavelengths.

In the SENSO project, two types of equipment were used:

- A robot with air-coupled transducers, developed by the French Central Engineering School of Lille (Ecole Centrale de Lille) [29] to automatically move non-contact sensors along the measurement profile.

- A robot developed by IFSTTAR-Nantes that uses a moving laser interferometer as a receiver [13].

\subsubsection{Non-contact robot}

Fig. 6 shows the robot used to generate and receive surface waves by varying the distance between the emitter and the receiver (Fig. 5). Two identical, air-coupled, angular transducers were positioned at the critical angle for Rayleigh wave generation and then moved incrementally, parallel to the surface of the specimens.

Due to significant energy losses (98\%) at the air/concrete interface, very long, amplified signals that change continuously in frequency ("chirp") need to be generated and received. The chirp varies in frequency over the range [50-300 kHz] and has a central frequency close to $150 \mathrm{kHz}$. This technique allows higher peak power levels to be achieved [30].

Wave emission, displacement of the transducers and signal processing are controlled by a computer. The signal recorded by the receiver can be described in time and frequency by the following equation:

$s(t, R)=\sum_{1}^{N} s_{i}\left(t, R_{i}\right)$ and $S(\omega(R)=\mathfrak{J}[S(t, R)]$

where $\mathfrak{I}$ is the Fourier transform, $t$ the time, $R$ the transmitter-receiver distance, and $\omega$ the pulsation.

Measurements made along a line with $N$ points (generally 20) designate a "profile", and the acquisition time for one profile is about $20 \mathrm{~min}$. The study frequency $f_{c}$ and the common frequency band were fixed. For the project described here, $f_{c}=150 \mathrm{kHz}$. Three parallel profiles were averaged, in order to minimise the influence of the concrete's spatial inhomogeneities.

The following parameters were calculated:

1 - For two distances $R_{1}$ and $R_{2}$, the dispersion characteristic of the surface waves as a function of frequency allows the phase velocity Écouter $V_{p h}(f)$ to be determined as:

$V_{p h}(f)=\frac{\omega\left(R_{2}-R_{1}\right)}{\varphi_{2}-\varphi_{1}}=\omega \frac{\Delta R}{\Delta \varphi(f)}$

in which $\Delta \varphi=\varphi_{2}-\varphi_{1}$ is the phase difference between the signals measured at two different observation points, where the difference in the source-to-observation point distances given by $\Delta R=R_{2}-R_{1}$, and $V_{p h}(f)$ is calculated using the Slant Stack transformation [31] [32].

Four distinct velocities are calculated [29]: the group velocity, using the time domain correlation technique, the group velocity derived from a linear regression of the maxima of the signals $s_{i}(t, R)$, the phase velocity at the central frequency $f_{c} V_{p h}\left(f=f_{c}\right)$ and the phase velocity at the frequency which produces a maximum value for the Slant Stack transformation. The velocity $V\left(f_{c}\right)$ is taken as the average of these four different velocities.

To take the concrete's inhomogeneities into account, $P=10$ parallel profiles were used. By averaging the values of $V\left(f_{c}\right)$ obtained over $P$ profiles, the following output ND parameters were obtained: the Mean Velocity of the Surface Wave: SW me (m/s), and its standard deviation, referred to as the Standard Deviation of the Mean Velocity of the Surface Wave: SW sd me (m/s).

The frequency-dependent attenuation coefficient $\alpha(\omega)$ is calculated for each pair of signals, recorded at distances $R_{i}, R_{j}[33]$ :

$\alpha(f)=\frac{1}{R_{i}-R_{j}}\left[10 \log \left(\frac{X_{i}(\omega)}{X_{j}(\omega)}\right)-10 \log \left(\frac{R_{j}}{R_{i}}\right)\right]$

The value of the attenuation $\alpha\left(f_{c}\right)$ at the central frequency and its standard deviation are obtained from the average of 8 signal characteristics, using a specific fit, with a theoretical variation in which $V$ is assumed to be constant [33]

$A(\alpha, R)=e^{-\alpha_{c} R} / \sqrt{R}$

By averaging $\alpha\left(f_{c}\right)$ over $P$ profiles, the output ND parameters obtained are: the Surface Wave Attenuation $\mathrm{SW}$ at $(\mathrm{dB} / \mathrm{m})$, and the Standard Deviation of the Surface Wave Attenuation SW sd-at $(\mathrm{dB} / \mathrm{m})$.

The attenuation $\alpha\left(\omega_{c}\right)$ is also expressed using the quality factor commonly used in Civil Engineering [3]

$Q\left(\omega_{c}\right)=\frac{\pi}{\alpha\left(f_{c}\right) \cdot \lambda_{c}}$

where $\lambda_{c}$ is the wavelength corresponding to the frequency $f_{c}$ under study. qf.

The ND parameter is the Quality Factor of the Surface Wave: SW

\subsubsection{Laser interferometer robot}

For this second development, the surface waves were generated with a piezoelectric wedge type of contact transducer [29] operating over the frequency range $[50 \rightarrow 250 \mathrm{kHz}$ ] and centred at $120 \mathrm{kHz}$. The waves were detected by a laser interferometer which measures a particle's velocity in a direction normal to the surface. 

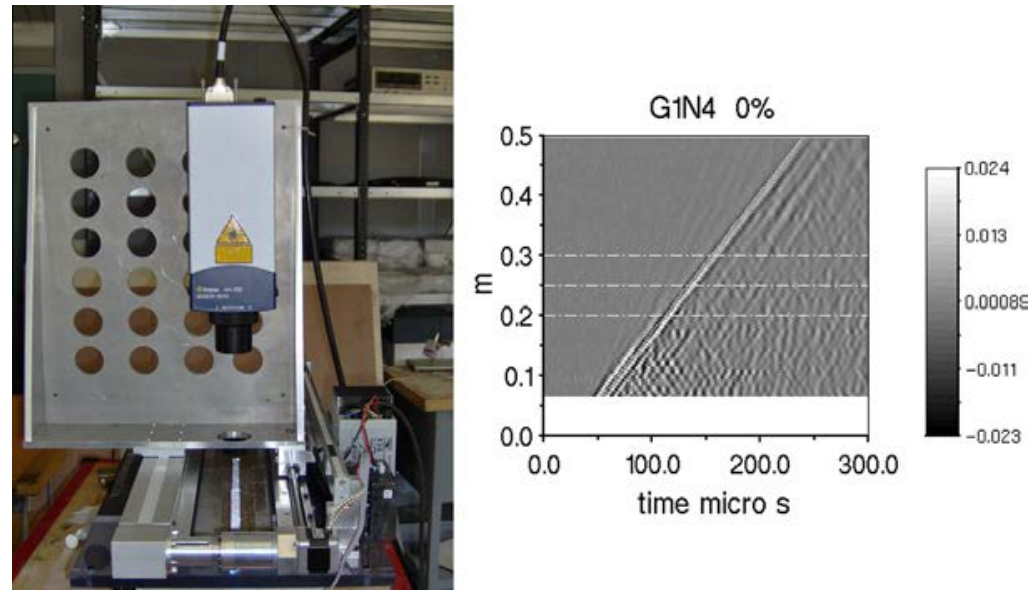

Fig. 7. Device for Laser measurement and a profile obtained on $43 \mathrm{~cm}$ of displacement.

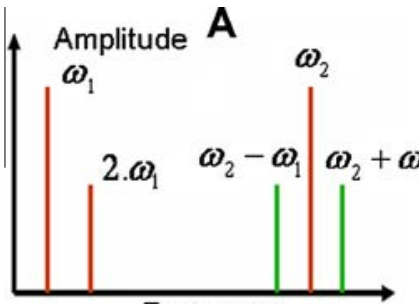

B

Frequency

Fig. 8. A spectrum of the transmitted wave with a nonlinear behaviour B Implementation of the technique on site.

A specific device was designed to monitor the displacement of the interferometer during measurements on the project slabs. The emitter was fixed to one end of the slab, and the interferometer was displaced along a line, allowing measurements to be made at $5 \mathrm{~mm}$ steps, over a total length of $430 \mathrm{~mm}$. The acquisition time needed for one profile was generally $30 \mathrm{~min}$.

The phase velocities were determined from the seismogram shown in Fig. 7, using the Slant Stack procedure in the frequency domain [31,32], based on signals recorded at distances ranging between $3 \mathrm{~cm}$ and $38 \mathrm{~cm}$ from the source, in order to avoid near-field effects [34]. The phase velocities corresponding to wavelengths: 1 , 2, 3, 4 and $5 \mathrm{~cm}$ were selected.

The ND parameters are the Surface Wave Velocity at different wavelengths $\lambda$ : SW $1 \mathrm{~cm}(\mathrm{~m} / \mathrm{s}), S W 2 \mathrm{~cm}(\mathrm{~m} / \mathrm{s}), S W 3 \mathrm{~cm}(\mathrm{~m} / \mathrm{s})$, and $\mathrm{SW} 4 \mathrm{~cm}(\mathrm{~m} / \mathrm{s})$. These correspond to frequencies ranging from approximately $50-250 \mathrm{kHz}$.

It was decided to extract the phase velocity from the dispersion curve as a function of wavelength, in order to investigate the material at a similar depth in all of the slabs. An apparent velocity was also calculated for each profile, using a linear regression based on the amplitude maxima of the signals. The apparent velocity is related to the group velocity, since it can be directly correlated with the signal's energy, via the amplitude of the main wave packet. The ND parameter is: SW ap (m/s).

Some other ND parameters specific to the attenuation measurements were also measured, but were found to have widely dispersed values. This can be explained by the fact that the averaging process is not sufficient to recover the attenuation properties of the equivalent homogeneous medium [13]. Using a process similar to that applied in the case of the non-contact robot, an apparent quality factor (Eq. (9)) was obtained from regression analysis of the amplitude maxima of the signals following the geometrical damping correction. The ND parameter is the Quality Factor of the apparent surface wave: SW qf ap.

The regression processes were applied in the frequency domain for two other quality factors, calculated for the wavelengths $1 \mathrm{~cm}$ and $2 \mathrm{~cm}$. The ND parameters are the Quality Factor of the Surface Wave at wavelengths $\lambda$ : SW qf $1 \mathrm{~cm}$ and $S W$ qf $2 \mathrm{~cm}$.

\subsection{Nonlinear acoustics}

The nonlinear behaviour of several materials is described by the equation

$\sigma=M_{0} \cdot \varepsilon\left(1+\beta n l \varepsilon+\delta n l \varepsilon^{2} \ldots\right)+\alpha n l(\varepsilon, \dot{\varepsilon})$

in which $\sigma$ is the stress, $\varepsilon$ the strain and $M_{0}$ the linear modulus of elasticity.

Nonlinear behaviour of the Landau-Lifshizt type [35] describes the nonlinearity of atomic bonds with the parameters $\beta \mathrm{nl}$ and $\delta \mathrm{nl}$. As proposed by the authors in [14], at the mesoscopic scale, the nonlinearity induced around networks of pores and microcracks by contact, cohesion/decohesion, capillarity phenomena etc., is represented by the phenomenological parameter $\alpha$ l.

Several techniques can be used to estimate the material's nonlinear behaviour [36]: harmonic generation [37], amplitude subtraction [38], resonant frequency shift [39-41], and frequency modulation $[42,43]$. In order to take the geometric constraints and experimental conditions into account, the wave's modulation was used. An example of crack excitation is presented in Fig 8: spectral analysis reveals the presence of the second harmonic $2 \cdot \omega_{1}$ and frequency modulation. The use of two sources emitting at different frequencies $\omega_{1}$ and $\omega_{2}$, where $\omega_{2} \gg \omega_{1}$, leads to modulation of the higher frequency $\omega_{2}$ by the lower frequency $\omega_{1}$ if 
there is any nonlinearity present in the material. The spectrum is then characterised by sidebands at the frequencies $\omega_{2} \pm \omega_{1}$, centred around $\omega_{2}$ as shown in Fig. 8A.

In the case of the specimens studied during our project, the lower frequency $\omega_{1}$ was generated, as in industrial situations, using the mechanical energy associated with structural resonances. An impact, exciting all of the slab's resonance modes, generated a low frequency with sufficient energy to activate the nonlinear elements included in the network of pores and cracks in the concrete. As the wave spectrum is very wide (all modes are sought), it is difficult to extract the amplitude of the sidebands. In this case, it has been shown [44] that $\alpha \mathrm{nl}$ is proportional to the ratio of the energy generated in the sidebands Energy $(S B)$ to that supplied to the sample at the low Energy $(L F)$ and high Energy $(H F)$ frequencies:

$\alpha n l \propto \frac{\operatorname{Energy}(S B)}{\operatorname{Energie}(L F) \cdot \operatorname{Energie}(H F)}$

The experimental protocol is illustrated in Fig. 9 [45], where the low frequency is generated by a $24.8 \mathrm{~g}$ ball dropped from a height of $20 \mathrm{~cm}$ onto the same point of each specimen.

A continuous sine wave $(250 \mathrm{kHz})$, modulated by the low frequency, is transmitted through the thickness of the slab and used to analyse the material. The excitation frequencies and the slab's response are recorded simultaneously.

The ND parameter is a dimensionless quantity referred to as the NonLinear Acoustic coefficient NLA.

\section{Classification of the ND parameters}

The aim of this section is to propose a classification, based on specific criteria, for the ND parameters. The ND parameters are listed and classified in Table 3, together with the quality estimators and classification of the measurements in the last three columns. In this section, the estimators are defined and the ND parameters are classified. This process allowed five ND parameters to be selected, and their dependence on the indicators to be studied.

\subsection{Methodology}

Two classification approaches are used.

In the first approach an index is proposed, reflecting the ability of the ND parameter to discriminate between useful information and noise. This quality index, QI, calculated from the variance of the measurements at different scales, is well suited to studies in the laboratory under controlled experimental conditions.

In the second approach, the variation of the measurements obtained from each batch was used: this variation coefficient, VC, represents the uncertainty of the measurements in a context of spatial variability, which can be compared to in situ measurements.

The latter technique allows different techniques to be compared, even if they do not generate the full set of data required to compute the QI.

The first approach analyses the variances at different scales: $V 1$, V2, V3 [46].

$V 1$ is the variance resulting from 10 identical measurements made at one point. It represents the variability of the measurement. $V 2$ is the variance resulting from measurements made at three different points on each slab. It represents the spatial variability of a slab. $V 3$ is the average of the variances taken over the eight slabs of each batch. It represents the spatial variability of the slab in a given batch.

The classification criterion used in this table is related to the quality index, which is defined as:

$\mathrm{QI}=-\log \frac{V_{1}}{V_{3}}-\log \frac{V_{2}}{V_{3}}$

The quality index QI increases when the variabilities $V 1$ and $V 2$ are low compared with V3. It demonstrates the good repeatability of the measurement in a localised, but spatially variable context. The ND parameter becomes more effective for the detection of changes in the material when the index increases. The last column of Table 3 indicates the classification given to the ND parameters, when they are ranked by decreasing quality index (QI). Some techniques were ranked at the bottom of the list $(\mathrm{QI}=-10)$ when insufficient variance data ( $V 1$ and $V 2$ ) was available.

The second approach to the classification of the ND parameters is based on the calculation of the variation coefficient VC:

$\mathrm{VC}=\sum_{i=1}^{8}\left(S D\left(N D p_{i}\right) / N D p_{i}\right)$

where a smaller VC coefficient corresponds to a more reliable measurement.

For the eight slabs in each batch $i$, and for each degree of saturation, the average value of the ND parameter $N D p_{i}$ and the mean standard deviation $S D\left(N D p_{i}\right)$ were defined, for all values of $i$. The variation coefficient allowed a new classification to be established, including the ND parameters with the lowest ranking in the previous classification, as a consequence of their poor quality index $(\mathrm{QI}=-10)$.

\subsection{Results}

The previous definition of the classification index allowed the most relevant ND parameters to be retained for the evaluation of the concrete slabs. The smaller the coefficient VC and the higher

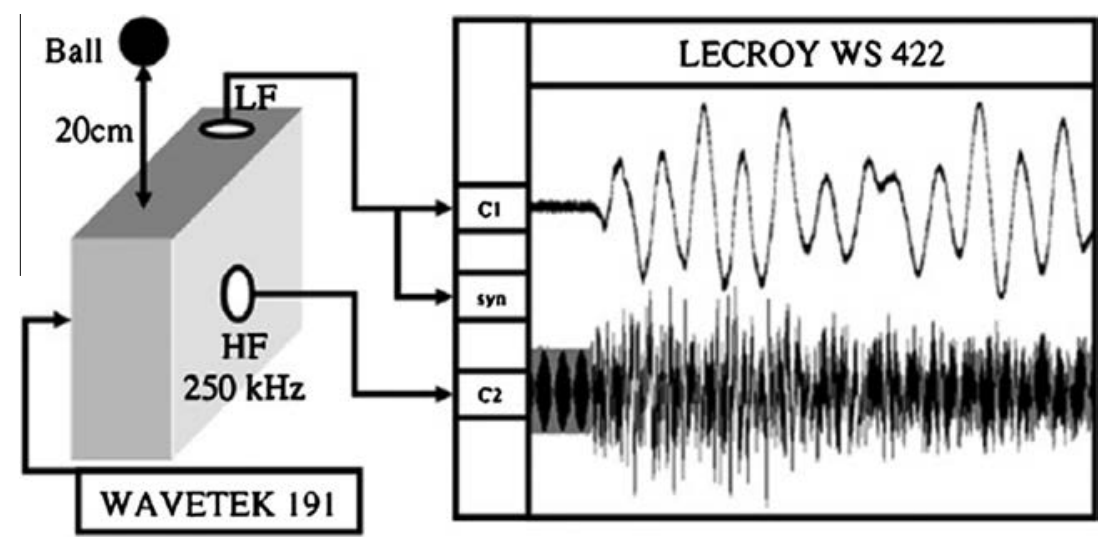

Fig. 9. Nonlinear acoustic parameter measurement principle. 
the quality index QI, the more reliable the measurement. The best results are generally ranked at the top of Table 3. This classification of the ND parameters can be analysed and explained as follows:

- With the exception of some specific cases, velocity measurement techniques have the best performance in terms of quality index and coefficient of variation. These can be found at the top of Table 3.

- The velocity calculated from the impact echo \#1 (PW IE) has the best QI, but not a very good VC. In practice, it is derived from a set of four equations and measurements, and the measurement errors are cumulative.

- Although the Impact Echo measurements often have the best Quality Index, they do not always have the best Variation Coefficient. The response is conditioned by the total volume of concrete, and the measurements do not depend on the local composition of each slab. The mechanical properties (Poisson's ratio and the modulus of elasticity) obtained with the Impact Echo technique do not have a satisfactory variation coefficient, even though they have a good quality index. As their value depends on the square of the measured ND parameter (frequency), the uncertainties increase accordingly.

- The reference measurement for the evaluation of the sound velocity is that based on the wave transmission at a central frequency of $250 \mathrm{kHz}$ \#5 (PW 250). Although it does not have the best quality index, its variation coefficient shows that it leads to reproducible results throughout the batch.

- Surface wave velocities are well ranked. It should also be noted that the surface wave velocities measured at a fixed wavelength (\#9 SW $1 \mathrm{~cm}-\# 7 \mathrm{SW} 2 \mathrm{~cm}-\# 4 \mathrm{SW} 3 \mathrm{~cm}-\# 3$ SW $4 \mathrm{~cm}-\# 8 \mathrm{SW} 5 \mathrm{~cm}$ ) lead to better results than the surface wave velocities obtained from a combination of frequencies, such as the mean (\#19 SW me) or apparent (\#21 SW ap) surface wave velocities. The latter two velocities are highly sensitive to gradients in the concrete's properties as a function of depth, and have varying influences at each wavelength. These two parameters are averaged. The suitability of the various techniques needs to be studied, in the context of the on site implementation capabilities.

- The ND parameters of surface wave velocity are related to the depth of penetration. The greater the wavelength, the greater the affected depths and, with respect to the size of the aggregates, the better the quality. This may be due to the fact that the slabs have near-surface inhomogeneities, the influence of which is averaged when the entire volume is considered.

- As the ND parameters associated with the measurements affecting the entire volume of the specimens (transmission at $250 \mathrm{kHz}$ PW 250 and impact echo PW IE) have the best quality index or variation coefficient, the volume affected by the wave should be carefully taken into account when designing experimental protocols and selecting ultrasound techniques.

- Although they are sensitive to changes in the material, attenuation, quality factor and nonlinearities lead to significant uncertainties, as shown by their high coefficient of variation and low quality index. Further technical developments are required before practical use can be made of these ND parameters. This has already been achieved in the case of several specific techniques $[21,47]$.

- In the following section, only the velocity measurements are taken into account, since in most applications they had a high ranking in our classification. These techniques are also comparable in terms of results and quality. The velocities (italicized in Table 3) obtained by impact echo \#1 (PW IE), surface wave \#4 (SW $3 \mathrm{~cm}$ ) and transmission \#5 (PW 250) were selected for the quality index. Despite their apparently mediocre performance, two other velocities were also selected (\#21 PW 50 and \#19 SW me) because they are derived from industrial or robotic techniques.

\section{Comparison of ND parameters versus the indicators}

In this section, the ND parameters derived from the ultrasonic measurements are compared, to determine whether they have similar responses to the indicators: porosity, degree of saturation, modulus and compressive strength. Five velocities, three pressure waves (PW IE, PW 250, PW 50) and two surface waves (SW $3 \mathrm{~cm}$, SW me), derived from different techniques and determined by three different partners, were selected using the same set of slabs. Only one ND parameter derived from each experimental device was selected. Their variation coefficients ranged between 1.6 and 3.1. These parameters have good Quality Indexes, with the exception of PW 50, for which not all of the data were provided. Table 4 lists the five selected ND parameters together with data (code, classification, frequency, wave' length, the partner that has developed the technique). We have three pressure waves.

The following observations can be made:

- The Impact Echo measurements affect the entire volume of the slab.

- The transmitted waves (PW 250 and PW 50) are propagated through the smallest thickness of the specimen $(12 \mathrm{~cm})$, and a volume of material, depending on the size of the transducers.

- The waves transmitted at $50 \mathrm{kHz}$ (PW 50) correspond to the Pundit type of industrial material.

- When surface waves are used, the investigated depth is approximately $3 \mathrm{~cm}$ for the SW $3 \mathrm{~cm}$ ND parameter, and approximately $1.5 \mathrm{~cm}$ for the SW gr ND parameter.

The data generated by this study was thus provided by a variety of techniques and different operators. The various techniques allow different volumes of concrete to be analysed. That can lead to difficulties, when the results of different experiments are compared.

In the first place the five selected ND parameters were compared for the cases of $0 \%$ and $100 \%$ saturation characterised by a large number of measurements. For these two degrees of saturation, they were compared as a function of the following indicators: porosity, modulus and strength.

Then the evolutions of the five ND parameters were studied as a function of the degree of saturation (0-30-50-70-100\%).

The analyses are limited to the six batches having the same aggregates (nature and size), but variable water to cement ratios hence different porosities and mechanical properties.

\subsection{Velocity dependence on porosity}

Variations of each ND parameter were compared in two stages.

First the variation of the five velocities as a function of porosity is shown in Fig. 10, for the case of a controlled degree of saturation equal to $100 \%$. For each batch, the porosity was measured with three cores [1] and a mean value and the standard deviation were calculated. The velocity and its standard deviation ( \pm 1 SD) were calculated for each of the ten slabs in the batch, and the average of these ten values was then plotted. The lowest values of the standard deviation were found for the PW 250 and SW $3 \mathrm{~cm}$ techniques. Both techniques have the best uncertainties because either they homogenise a large volume of material or they affect a depth that remains constant. The other techniques are less accurate because they are more difficult to implement or the calculations involve several measurements, each of which has its own specific errors. 
Table 4

Selected ND parameters for comparison: CLassification, Code, Frequency, Wave Length and Partners that have measured.

\begin{tabular}{|c|c|c|c|c|c|}
\hline Velocity $(\mathrm{m} / \mathrm{s})$ determined by & $\mathrm{CL}$ & Code & $f(\mathrm{~Hz})$ & $\lambda(\mathrm{cm})$ & Partner \\
\hline Impact echo : calculated velocity & 1 & PW IE & 20 & 20 & IFSTTAR \\
\hline Surface Wave $\lambda=3 \mathrm{~cm}$ : laser measurement & 4 & $\mathrm{SW} 3 \mathrm{~cm}$ & 70 & 3 & IFSTTAR \\
\hline Transmission: central frequency $250 \mathrm{kHz}$ & 5 & PW 250 & 250 & 1.8 & LMA \\
\hline Surface Wave Group velocity: air transducers measurement & 19 & SW me & 150 & 1.2 & $\mathrm{ECL}$ \\
\hline Transmission: central frequency $50 \mathrm{kHz}$ & 21 & PW 50 & 50 & 9 & $\mathrm{ECL}$ \\
\hline
\end{tabular}

Secondly for the two degrees of saturation, $100 \%$ and $0 \%$, although the bulk velocities plotted in Fig. 11 could be expected to be identical, nearly parallel, although shifted, curves are obtained. The same remarks can be made for both of the techniques relying on the use of surface waves.

These differences can be explained by the following factors:

- the measurements were not made at the same time by all the partners. The material properties may have changed between these different times,

- the surface waves affect different depths of the cover concrete, which is dispersive as a result of gradients in the material's composition, and a possible gradient in the degree of saturation, as a function of depth. The phase velocity $(\mathrm{SW} 3 \mathrm{~cm})$ and the group velocity (SW me) have different wavelengths, and different contributions from the concrete are averaged. This leads to different calculated velocities,

- the velocities obtained for pressure waves transmitted through the slab (PW 250) are higher than those measured by the Pundit (PW 50) and the Impact Echo (PW IE) techniques. The two methods, PW 50 and PW IE, give very similar results, with velocities obtained at lower frequencies (between 10 and $50 \mathrm{kHz}$ ) than those used in the transmission method $(250 \mathrm{kHz})$. The observation of greater velocities at higher frequencies is related to the material's dispersion [17].

This is confirmed by the homogenisation models [48,49], when implemented for the case of spherical scatterers used to approximate the aggregates. A decrease in the measured velocity of the ultrasonic waves can be expected for frequencies below $150 \mathrm{kHz}$, and this behaviour was confirmed by experimental results [50].
This frequency dependence is the same for both degrees of saturation, because the wave frequencies are identical.

All of these results can be described with regression laws, in order to compare the slopes, which indicate the sensitivity, with the porosity indicator shown in Table 5 . The observed linear trend corresponds to a relationship of the type $V=a \cdot P+V_{O}$, where $P$ is the porosity and $a$ and $V_{O}$ are constants, similar to those found in the case of mortar [20]. The distribution of the experimental points with respect to this linear regression is expressed by the determination coefficient $r^{2}$. The extreme porosity values correspond to extreme values of Young's modulus and strength (G1 and G8).

In the case of a controlled degree of saturation equal to $100 \%$, the slopes $a$ and equation constants $V_{O}$ are very similar for all transmission wave excitations, as well as for the two types of surface wave. The correlations are all similar, with a good coefficient $r^{2}$, except for the case of the Mean Velocity of Surface Wave (SW $\mathrm{me}$ ): the non-contact robot was under development during these measurements, and did not allow the signals to be recorded with an adequate signal-to-noise ratio. The slopes of the surface wave techniques are twice as small as that of the transmission technique. This ratio corresponds to the ratio of the velocities. It should be noted that for the case of $0 \%$ degree of saturation, only two correlations emerge with a determination coefficient above 0.9 , because it is far more difficult to achieve and maintain the degree of saturation at $0 \%$ than at $100 \%$.

\subsection{Velocity dependence on elasticity modulus}

The variations in velocity as a function of the modulus of elasticity are plotted in Fig. 12A for the PW 250 tests only, in order to present the uncertainties corresponding to $0 \%$ and $100 \%$ degree
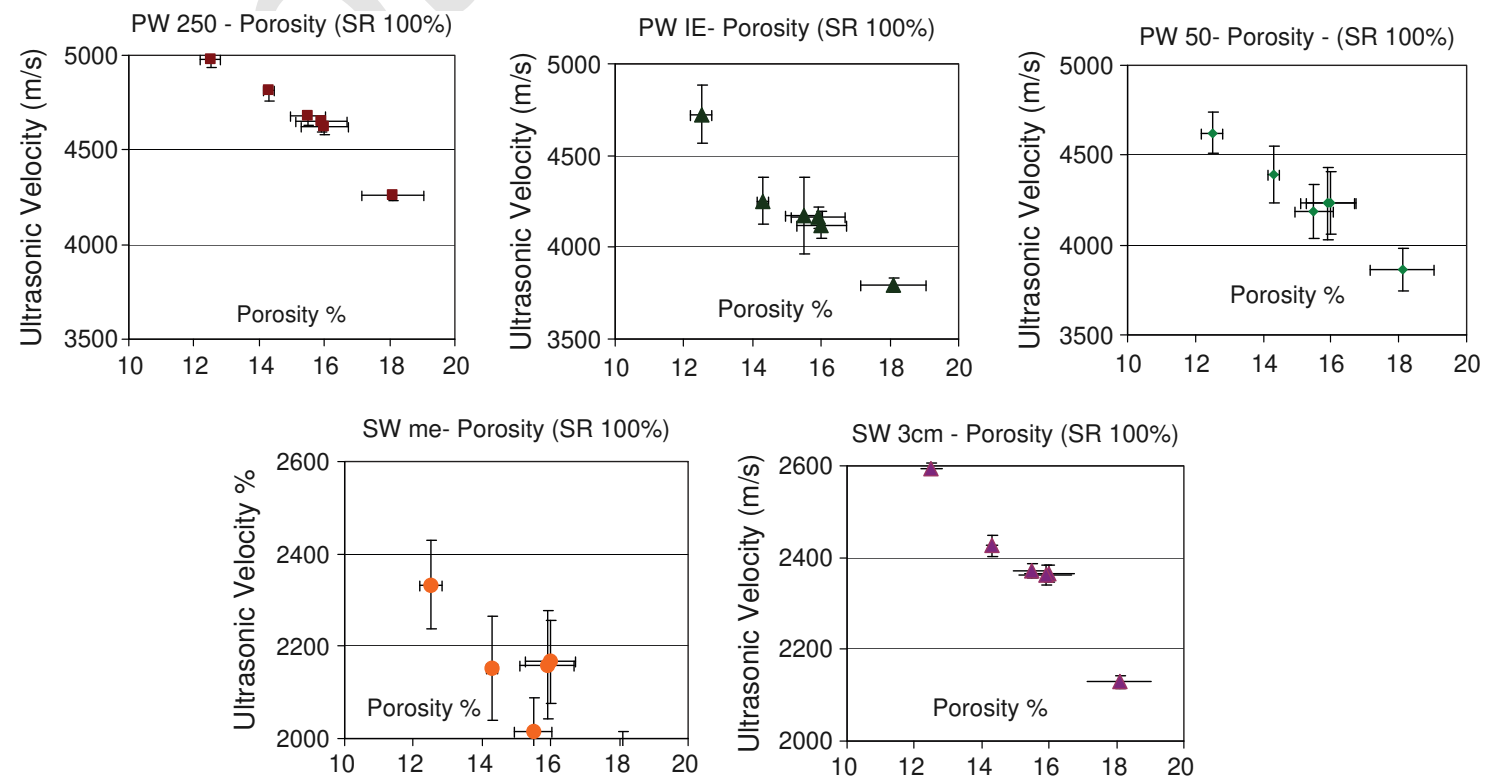

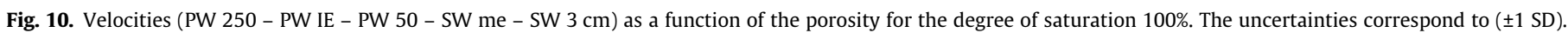



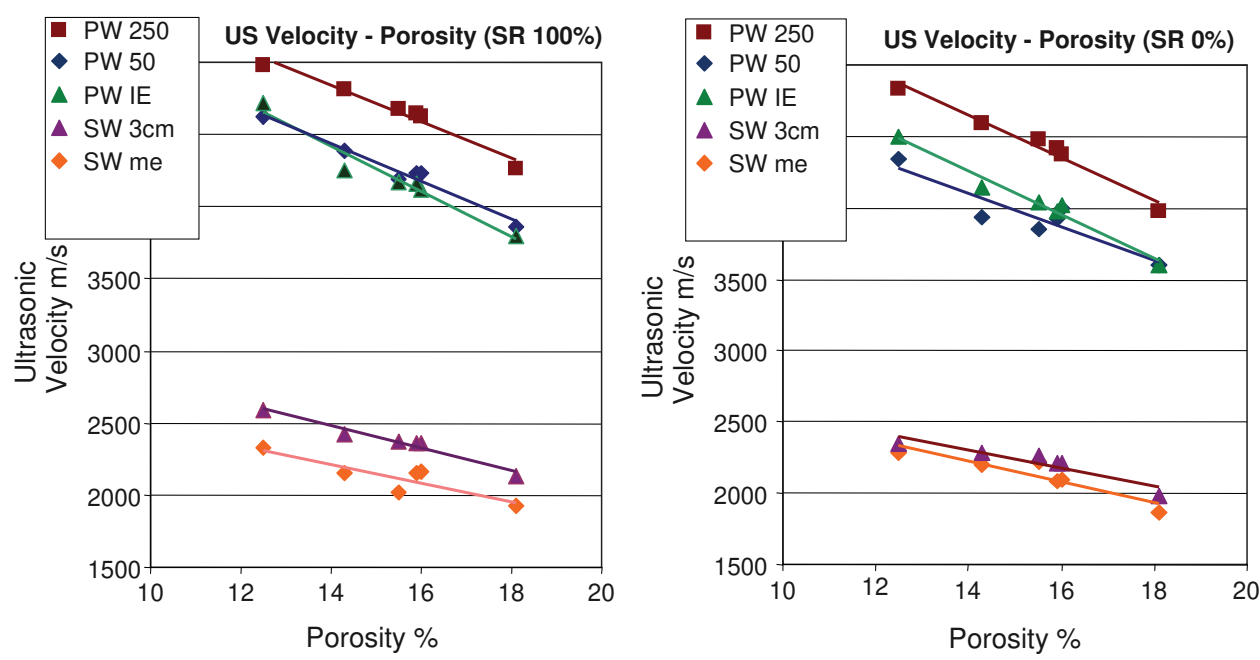

Fig. 11. Linear regression between ultrasonic velocities and porosity for two degrees of saturation: $100 \%$ and $0 \%$.

Table 5

Linear regression of ND parameter (velocity $\mathrm{m} / \mathrm{s}$ ) as a function of the porosity (Por \%) for the degrees of saturation $100 \%$ and $0 \%$.

\begin{tabular}{llllll}
\hline ND parameter & Degree of saturation & & \multicolumn{2}{l}{$\begin{array}{l}\text { Determination } \\
\text { coefficient } R^{2}\end{array}$} \\
\cline { 2 - 3 } \cline { 5 - 6 } Velocity $(\mathrm{m} / \mathrm{s})$ & $100 \%$ & $0 \%$ & & $100 \%$ & $0 \%$ \\
\hline PW 50 & $6286-132.1 *$ Por & $5740-116.9 *$ Por & & 0.969 & 0.837 \\
PW 250 & $6602-125.6 *$ Por & $6748-149.6 *$ Por & & 0.961 & 0.971 \\
PW IE & $6613-156.6 *$ Por & $6398-152.9 *$ Por & & 0.953 & 0.974 \\
SW me & $3137-65.8 *$ Por & $3242-72.6 *$ Por & & 0.754 & 0.845 \\
SW 3 cm & $3578-78.2 *$ Por & $3180-62.7 *$ Por & & 0.959 & 0.865 \\
\hline
\end{tabular}

of saturation. These are identical for the ultrasonic measurements described in the case of the porosity analysis. On the other hand, the modulus of elasticity uncertainties are derived from three compression tests. These uncertainties can be significant and pose the problem of the representation and the quality of the mechanical tests.

Fig. 12B shows the variation of the five velocities as a function of the modulus of elasticity. It is of interest to observe the distribution of the modulus data: most of the data points are centred around the Young's modulus value of $30,000 \mathrm{Mpa}$. The two extreme values $(21,000$ and $38,000 \mathrm{MPa})$ that correspond to the cases of the most and least porous concretes are very far from this central value. A small variation or error in the measurement of these velocities can have a strong influence on the regression laws derived for the five ND parameters.

Concerning the regression laws provided in Table 6, which are used to describe the velocity as a function of Young's modulus, the same remarks can be made as in the case of the porosity. In each group of techniques (transmission or surface waves), the slopes are very similar and three of these techniques merge in terms of coefficients of determination (PW 250 - PW IE - SW $3 \mathrm{~cm}$ ). The lower values of these coefficients correspond to surface waves.

\subsection{Velocity dependence on strength}

The variations in ultrasonic velocity as a function of the concrete's compressive strength are shown in Fig. 13A and B. This information is of considerable interest to the engineer. The same observations can be made as for the study of the modulus of elasticity. Once again, there are very significant uncertainties in the compression tests, such that the quality of these measurements is an
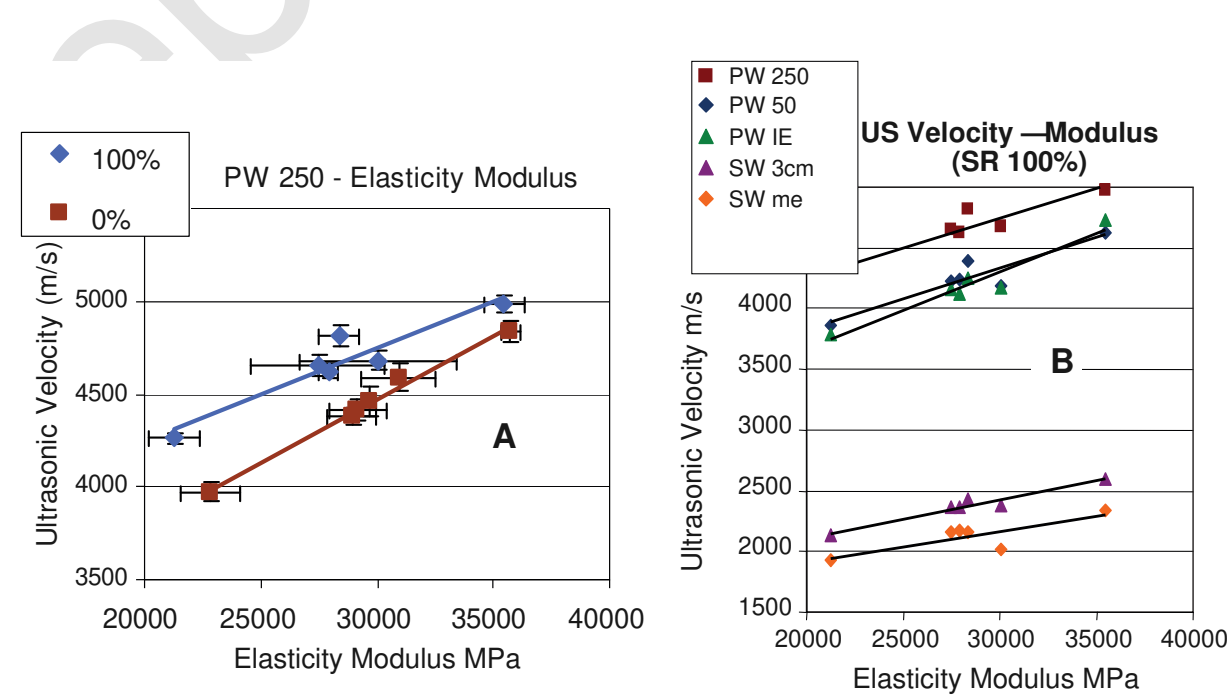

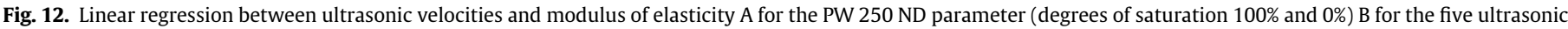
ND parameters (degree of saturation $100 \%$ ). 
Table 6

Linear regression of ND parameters (velocity $\mathrm{m} / \mathrm{s}$ ) as a function of the elastic modulus ( $E \mathrm{MPa}$ ) for the degrees of saturation $100 \%$ and $0 \%$.

\begin{tabular}{|c|c|c|c|c|}
\hline \multirow{2}{*}{$\begin{array}{l}\text { ND parameter } \\
\text { Velocity }(\mathrm{m} / \mathrm{s})\end{array}$} & \multicolumn{2}{|c|}{ Degree of saturation } & \multicolumn{2}{|c|}{ Determination coefficient $R^{2}$} \\
\hline & $100 \%$ & $0 \%$ & $100 \%$ & $0 \%$ \\
\hline PW 50 & $2806+0.051 * E$ & $2324+0.0547 * E$ & 0.862 & 0.898 \\
\hline PW 250 & $3265+0.0494 * E$ & $2428+0.0683 * E$ & 0.887 & 0.988 \\
\hline PW IE & $2403+0.0634 * E$ & $1983+0.0698 * E$ & 0.933 & 0.991 \\
\hline SW me & $1394+0.0257 * E$ & $1132+0.0336 * E$ & 0.686 & 0.885 \\
\hline $\mathrm{SW} 3 \mathrm{~cm}$ & $1474+0.0317 * E$ & $1354+0.0292 * E$ & 0.941 & 0.916 \\
\hline
\end{tabular}
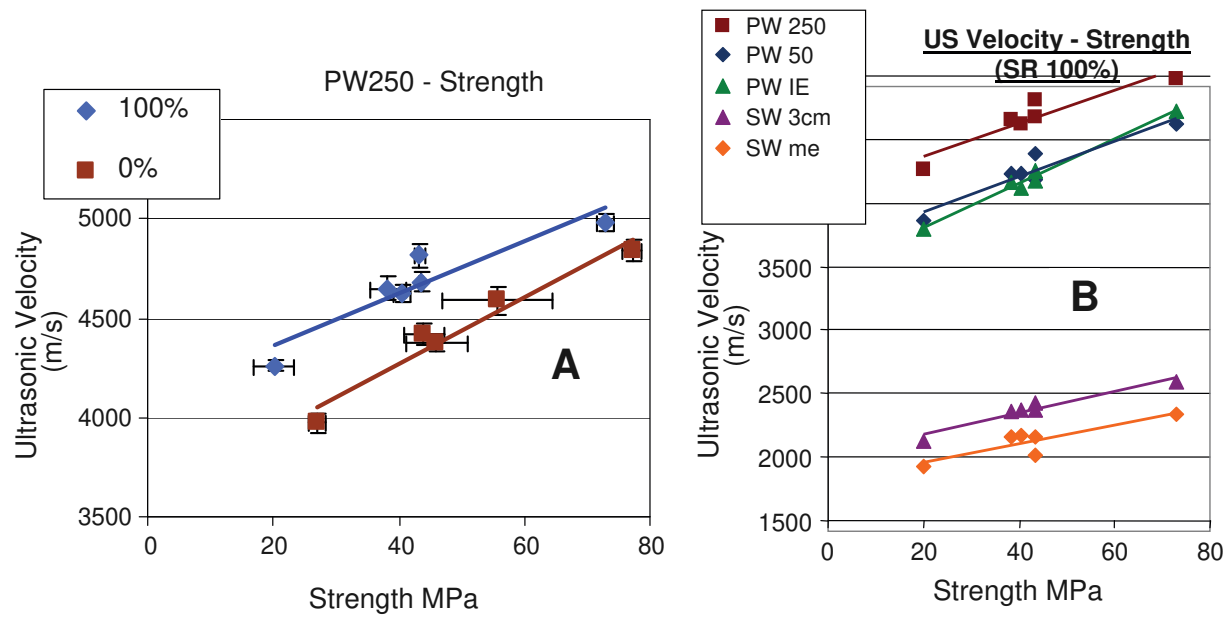

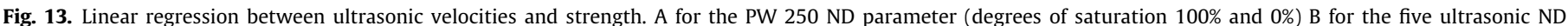
parameters (degree of saturation $100 \%$ ).

issue, which remains to be clarified. Although the regression laws appear to be well correlated, as shown by the coefficient of determination given in Table 7, these values have non-negligible errors, and considerable difficulties could be expected when using such ultrasonic measurements to determine the concrete's strength.

As for the modulus analysis, the regression law constants are very similar for each group of techniques (transmitted and surface waves).

\subsection{Velocity dependence on the degree of saturation}

Whereas previous analyses have focused on only two cases of saturation, in order to study the sensitivity and accuracy of each ND parameter as a function of the indicators, the experimental protocol was designed to include tests at intermediate degrees of controlled saturation. It was thus possible to test the velocity variations as a function of the degree of free water saturation in the porous network. In this aspect of the study, data for the ND parameters: PW 250 - PW IE - SW me - SW $3 \mathrm{~cm}$ was recorded.

The variations of two velocities (PW 250 - PW IE) as a function of the degree of saturation are shown in Fig. 14. Each concrete is characterised by its batch number and porosity ratio (G1 $12.5 \%$ G2 14.3\% - G3 15.5\% - G3a 16\% - G7 15.9\% - G8 18.1\%).

The variations are quite similar for both velocities. The PW 250 measurements are always about 10\% higher than the PW IE values. This confirms a previous comment concerning the velocity's sensitivity to frequency.

It can be seen that two quite different trends are present.

The first corresponds to the G8 batch, showing an increase and a maximum in the variation of the velocity as a function of degree of saturation $(70 \%)$.

The second trend indicates a fall in velocity at intermediate degrees of saturation. The amplitude of this fall is very small for the G1 concrete, which is a low porosity concrete. On the other hand, for specimens with a higher porosity, the velocity falls by approximately $20 \%$ before increasing again at higher values of degree of saturation. This specific behaviour has been observed for the cases of sandstone and rock [51,52]. The wave velocity is a function of the ratio between the Young's modulus and the density. When the concrete's degree of saturation increases, the modulus and density both increase, but the Young's modulus appears to be the dominant effect. Meanwhile, at very low saturations, the velocities are higher than at about $40 \%$ saturation. This phenomenon could

Table 7

Linear regression of ND parameters (velocity $\mathrm{m} / \mathrm{s})$ as a function of the strength $\left(f_{c} \mathrm{MPa}\right)$ for the degrees of saturation $100 \%$ and $0 \%$.

\begin{tabular}{|c|c|c|c|c|}
\hline \multirow{2}{*}{$\begin{array}{l}\text { ND parameter } \\
\text { Velocity }(\mathrm{m} / \mathrm{s})\end{array}$} & \multicolumn{2}{|c|}{ Degree of saturation } & \multicolumn{2}{|c|}{ Determination coefficient $R^{2}$} \\
\hline & $100 \%$ & $0 \%$ & $100 \%$ & $0 \%$ \\
\hline PW 50 & $3650+14.00 * f_{c}$ & $3376+13.58 * f_{c}$ & 0.896 & 0.916 \\
\hline PW 250 & $4104+13.09 * f_{c}$ & $3597+16.85 * f_{c}$ & 0.858 & 0.953 \\
\hline PW IE & $3448+17.55 * f_{c}$ & $3172+17.53 * f_{c}$ & 0.985 & 0.987 \\
\hline SW me & $1809+7.32 * f_{c}$ & $1698+8.18 * f_{c}$ & 0.767 & 0.911 \\
\hline $\mathrm{SW} 3 \mathrm{~cm}$ & $2008+8.51 * f_{c}$ & $1857+7.00 * f_{c}$ & 0.935 & 0.864 \\
\hline
\end{tabular}



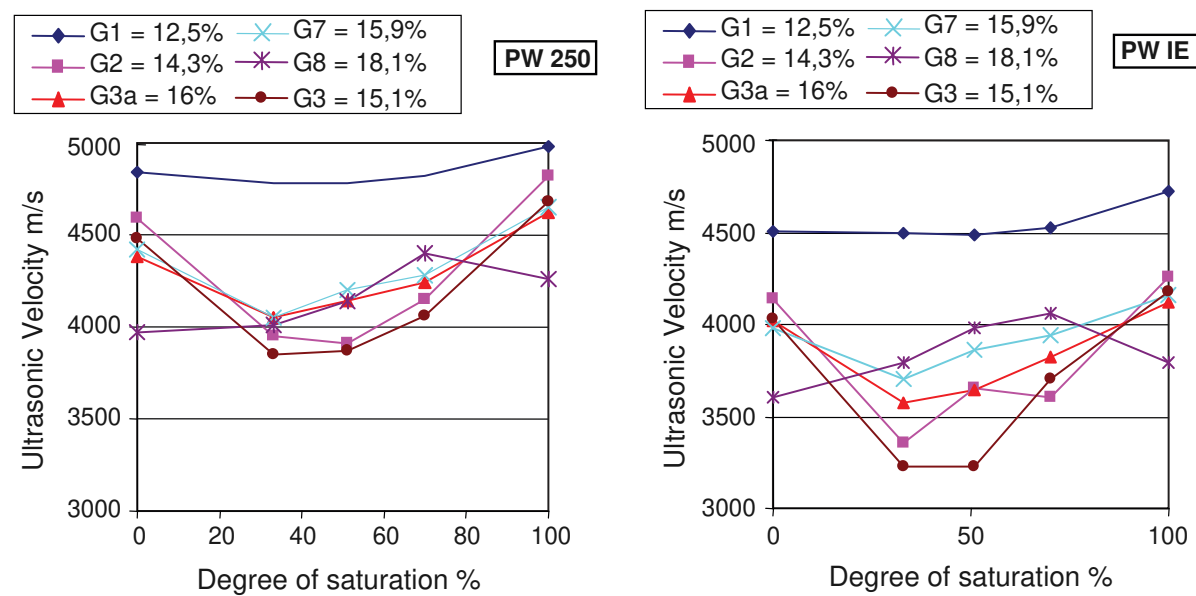

Fig. 14. Ultrasonic velocities PW 250 and PW IE versus the degree of saturation for the six batches.
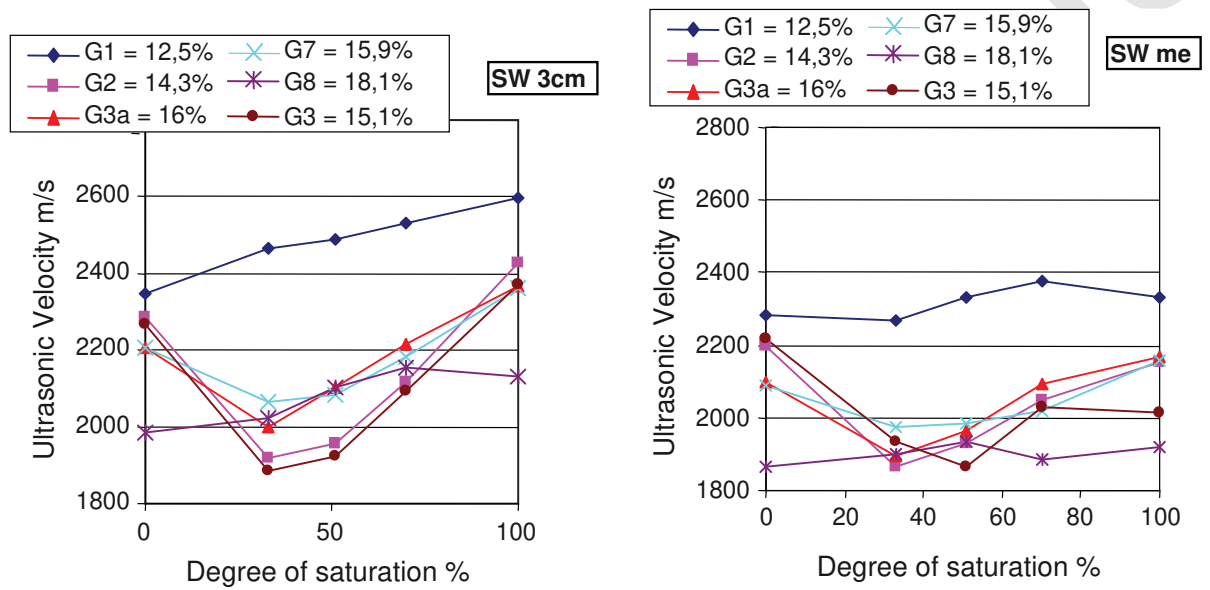

Fig. 15. SW $3 \mathrm{~cm}$ and $\mathrm{SW}$ me ultrasonic velocities versus the degree of saturation for the six batches.

be explained by a higher modulus, due to the presence of strong capillary forces $[27,51,52]$.

In the case of the G8 batch, which is very porous, another explanation can be given: the experimental campaigns lasted for more than 18 months, and concrete carbonation may have occurred over the 1 year period between the dry and intermediate degrees of saturation, which could have increased the velocities of the intermediate states.

In this context, if the behaviour at $0 \%$ saturation is not taken into account, a linear correlation can be established between the ultrasonic wave velocity and the degree of saturation. In practice, $0 \%$ is not a realistic value for the purposes of on site analysis. For the study reported here, it was agreed to work with realistic saturations only, lying in the range between $40 \%$ and $100 \%$.

The variations of the $S W 3 \mathrm{~cm}$ and SW me surface wave velocities under the same conditions are shown in Fig. 15. Again, the two devices used to measure the surface waves recorded similar variations. However, in the case of $0 \%$ saturation, the velocities measured by the two techniques were very similar, since under these conditions there is no water in the concrete and thus no gradient as a function of depth. Otherwise, in the case of intermediate and saturated degrees of saturation, the measured velocities were lower for the SW me. It strongly integrates the gradient in the measurement. Any gradient in the concrete's properties leads to additional difficulties, when it comes to measuring the ultrasound velocity. The use of only one fixed wavelength improves the quality of the results.

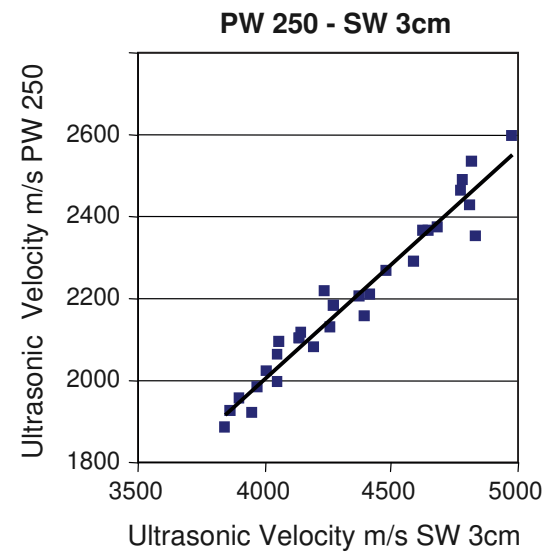

Fig. 16. Correlation between ultrasonic velocities (PW 250 versus $S W 3 \mathrm{~cm}$ ) for all the degrees of saturation.

It is also interesting to note the particularity in the behaviour of the concrete from the $\mathrm{G} 1$ batch, by comparing the two sets of data shown in Figs. 14 and 15. In the case of surface waves, the curves do not appear to have a minimum for G1. This is probably due to the volume affected by the waves, which differs from one technique to another. In addition, it is difficult to control the saturation as a function of depth, for slabs with a low porosity. In the case of 
Table 8

Cross correlation laws between ND parameters (velocities $\mathrm{m} / \mathrm{s}$ ) for all the degrees of saturation ratios and for the six batches.

\begin{tabular}{lll}
\hline ND parameter & Equation & $R^{2}$ \\
\hline PW 250 & $712+1.070 *($ PW IE $)$ & 0.901 \\
PW 250 & $237+0.559 *($ SW $3 \mathrm{~cm})$ & 0.951 \\
PW IE & $298+0.482 *($ SW $3 \mathrm{~cm})$ & 0.895 \\
SW me & $115+1.121 *($ SW $3 \mathrm{~cm})$ & 0.816 \\
PW 250 & $303+0.404 *($ SW me $)$ & 0.764 \\
\hline
\end{tabular}

the G1 concrete, it is difficult to homogenise the degree of saturation. The presence of water distribution gradients as a function of depth locally modifies the properties affecting the velocity of the ultrasonic surface wave. As a consequence, significant differences can be observed in measurements based on the use of surface waves for materials with a water content gradient, and those obtained by volume wave transmission.

A global comparison of the results can be made using a cross correlation, as shown for example in Fig. 16. All of the results are summarised in Table 8. The determination coefficient of the correlation allows the velocities to be compared in pairs.

The best combination is that including the two velocities PW 250 and SW $3 \mathrm{~cm}$ (Fig. 16). This pair also has the best Variation Coefficients, as can be seen in Table 8 . This indirectly confirms the validity of the VC as a classification tool for the quality of the ND parameters. The slope of this correlation is 0.559 , corresponding to the ratio between the transmission wave and surface wave velocities.

The slope of the correlation between the two surface waves generated by the two robots should be equal to 1 , whereas the experimentally determined value was 1.12 . This confirms that there is an absolute difference between $S W 3 \mathrm{~cm}$ (phase velocity) and SW me (association of group velocity and phase velocities), which are defined in different ways and characterise different depths (respectively 30 and $15 \mathrm{~mm}$ ). In addition, it appears that the depth characterised by the surface wave should be chosen to be as great as possible.

\section{Conclusions}

The techniques used during the SENSO project have on the one hand led to the development of new, original methods for ultrasonic measurements, and on the other hand promoted the study of the applicability and relevance of ultrasonic techniques for the investigation of concrete. Our conclusions are related to three main points: the technical input related essentially to the project, the progress achieved in the study of concrete, which will enrich future research work, and emerging studies.

- The most significant technical contribution of this study is the development of concrete investigation techniques based on the use of ultrasonic waves. Our research into wave propagation led to the proposal of solutions for the measurement of ND parameters related to different scales of the microstructure, which can be used to determine the material's sustainability indicators. Robots using air coupled transducers or lasers, designed for the study of surface wave propagation, have been proposed. The analysis of resonance frequency measurements using the Impact Echo technique has also allowed the mechanical characteristics of the concrete to be determined.

- The progress achieved in techniques for the analysis of concrete, which is likely to enrich future research efforts, can be described in terms of three different aspects: measurements results -classification of the techniques.
Concerning the measurements, the importance of knowing which zones are affected by the propagating wave has been demonstrated. The Rayleigh waves affect weak depths, and are sensitive to the gradients of concrete properties in this dimension. On the other hand, waves propagating through a slab average out the material's properties, and are only slightly sensitive to such gradients. The importance of the working frequency and its relationship with the diffusion phenomenon has also been demonstrated. In concrete, the velocity of sound increases with frequency, and in the case of surface waves it is important to work with a fixed frequency. It has also been shown that the phase velocity is more accurate than the Mean Velocity of the Surface Wave.

Concerning the results, it has been shown that the propagation velocities, measured using various different techniques, all have similar variations as a function of the different sustainability indicators. There are differences in the absolute value of the measured velocities, however these have been explained by the choice of wave frequency and the likelihood of local gradients in the material properties. It has been observed that variations in velocity as a function of the material's degree of saturation correspond to a particular case, and for future studies it is proposed to limit the material's degree of saturation to the range between $40 \%$ and $100 \%$.

Concerning the classification, those techniques which currently lead to the most reproducible results can been identified through the Quality Index and the Variation Coefficient. The spatial variability of the concrete is taken into account to quantify these two estimators. The pressure wave (PW 250) and Impact Echo wave (PW IE) transmission methods are confirmed in their role as reference techniques. The recently developed surface wave techniques are able to produce measurements with the same degree of reproducibility, if the phase velocity is measured at a high working frequency $(\mathrm{SW} 3 \mathrm{~cm})$.

- Finally, the three potential areas of concrete evaluation research, which emerge from this study are:

- New methods should be developed to investigate the relationship between attenuation, nonlinear behaviour and the physics of the concrete. Energy focalisation by temporal reversing, and the analysis of coda and backscattered waves are important research themes. It will also be useful to develop techniques for the measurement of gradients in the material (e.g. carbonation, degree of saturation) as a function of the distance below the surface.

- Wave propagation modelling will allow parametric studies to be developed, and signal patterns and their variations, as a function of concrete ageing, to be predicted. The analytical models which have already been tested have limited applications, because it is very difficult to accurately represent the complexity of the material. Numerical methods are likely to lead to promising solutions.

- In order to transfer ultrasonic techniques to in situ testing applications, the local material and environmental conditions will need to be assessed, in order to modify the regression laws between the ND parameters and the indicators, as shown by the results of the SENSO project. Moreover, the quality of the correlations generally depends on the quality of the ND parameter measurements, as well as the indicator measurements. The regression laws cannot be generalised to all types of concrete, without applying various modifications in order to take the environmental conditions and material composition into account. Two new on-going French projects: "Analysis and Capitalization for the Diagnosis of Constructions" and "Non-destructive evaluation of structures for damage prediction and optimisation of the follow-up" implement 
methodologies designed to make use of such corrections, for the on-site diagnosis of concrete. Finally, concerning the transfer of ultrasound technologies, it is important to bear in mind the ease or difficulty with which these techniques can be implemented on site. Transmission and resonant measurements are generally straightforward to implement, but depend on the geometry and thickness of the structure. It is possible to measure the surface wave velocity with a laser-assisted robot, or air-coupled transducers, and these measurements are reproducible. At this stage of development, the laser device is the most suitable technique for laboratory measurements, and the non-contact robot is the best option for on-site testing. Measurements made with the latter technique have a low signal-to-noise ratio, and thus have greater uncertainties. Their sensitivity to local vibrations and the surface conditions need to be introduced in future research.

The authors are confident that these three lines of research will lead to new and useful developments. Numerous methods and techniques, which are currently being deployed for in situ measurements, have been developed. The selection of the best solution for a given industrial problem will depend not only on the performance of the available techniques, but also on the context of the required tests and measurements.

\section{Acknowledgements}

The authors wish to thank the French National Research Agency (ANR) for their assistance during the SENSO project, and the MEDDTL French Ministry and the RGCU French Network for their support of the current ACDC project. MEDDTL (Ministry for the environment, sustainable development, transport and housing) RGCU (Civil Engineering and Urban French Network).

\section{References}

[1] Balayssac JP et al. Description of the general outlines of the French project SENSO - quality assessment and limits of different NDT methods. Construct Build Mater 2012;35:131-8.

[2] EN 12504-4 Testing concrete in structures - Part 4: determination of ultrasonic pulse velocity; 2004

[3] Standard Test Method for Pulse Velocity through Concrete; 2009.

[4] Breysse D, Abraham O. Guide méthodologique de l'évaluation non destructive des ouvrages en béton armé. Paris: Presses ENPC; 2005.

[5] Guyer RA, Johnson PA. Nonlinear mesoscopic elasticity; the complex behaviour of granular media including rocks and soil. GmbH\&Co, KGaA: WILEY-VCH Verlag; 2009.

[6] Vanaverbeke S, Van Den Abeele K. Two-dimensional modeling of wave propagation in materials with hysteretic nonlinearity. J Acoust Soc Am 2007;122(1):58-72.

[7] Gusez V, Tournat V, Castagnède B. In: Bruneau M, Potel C, editors. Materials and acoustics handbook. ISTE - WILEY; 2009. p. 431-66.

[8] Zhang Y, Abraham O, Larose E, Planes T, Le Duff A, Lascoup B, et al. Following stress level modification of real size concrete structures with Coda Wave Interferometry (CWI). Review of Progress in Quantitative Non Destructive Evaluation; 2010; 30; ISBN: 978-0-7354-0888-3.

[9] Payan C, Garnier V, Moysan J, Johnson PA. Determination of third order elastic constants in a complex solid applying coda wave interferometry. Appl Phys Lett 2009;94

[10] Waterman PC, Truell R. Multiple scattering of waves. J Math Phys 1961;2(4):512-37.

[11] Tourin A, Fink M, Derode A. Multiple scattering of sound. Topical review. Waves Random Media 2000;10(4):31-60.

[12] Chaix JF, Garnier V, Corneloup G. Ultrasonic wave propagation in heterogeneous solid media: theoretical analysis and experimental validation. Ultrasonics 2006;44(2):200-10.

[13] Chekroun M, Le Marrec L, Abraham O, Durand O, Villain G. Analysis of coherent surface wave dispersion and attenuation for non-destructive testing of concrete. Ultrasonics 2009;49(8):743-51.

[14] Guyer RA, Johnson PA. Nonlinear mesoscopic elasticity: evidence for a new class of materials. Phys Today 1999;52(4):30-6.

[15] Ostrovsky LA, Johnson PA. Dynamic nonlinear elasticity of geomaterials. Rivista Nuevo Cimento 2001;24(7):1-46.
[16] Projet ANR: Stratégie d'Evaluation Non destructive pour la Surveillance des Ouvrages en béton, Rapport; 2009.

[17] Aki T, Richards PG, editors. Quantitative seismology: theory and methods. Freeman; 1980.

[18] Popovics JS, Abraham O. Surface wave techniques for evaluation of concrete structures. In: Maierhofer G, Reinhardt HW, Dobmann G, editors. Nondestructive evaluation of reinforced concrete structures, vol. 2. Woodhead Publishing Ltd.; 2010. p. 441-65.

[19] Lu L, Chekroun M, Abraham O, Maupin V, Villain G. Mechanical properties estimation of functionally graded materials using surface waves recorded with a laser interferometer. NDT\&E 2011;44(2):169-77.

[20] Goueygou M, Lafhaj Z, Soltani F. Assessment of porosity of mortar using ultrasonic Rayleigh waves. NDT\&E Int 2009;42(8):353-60.

[21] Chekroun M, Le Marrec L, Abraham O, Durand O, Villain G. Analysis of coherent surface wave dispersion and attenuation for non-destructive testing of concrete. Ultrasonics 2009;49(8):743-51.

[22] Saniie J, Wang T, Bilgutay NM. Statistical evaluation of backscattered ultrasonic grain signals. J Acoust Soc Am 1988;84(1):400-8.

[23] Chaix JF, Garnier V, Corneloup G. Concrete damage evolution analysis by backscattered ultrasonic waves. NDT\&E Int 2003;36(7):461-9.

[24] Carino NJ, Sansalone M, Hsu N. Flaw detection in concrete by frequency spectrum analysis of impact-echo waveforms. Int Adv Nondestruct Test 1986;12:117-46.

[25] Sansalone MJ, Streett WB. Impact echo: non-destructive evaluation of concrete and masonry. Bullbrier Press 1997.

[26] Gibson A, Popovics J. Lamb wave basis for impact-Echo method analysis. J Eng Mech 2005;131(4):438-43.

[27] Villain G, Le Marrec L, Rakotomanana L. Determination of the bulk elastic moduli of various concretes by resonance frequency analysis of slabs submitted to impact echo. Eur J Environ Civil Eng 2011;15(4):601-17.

[28] Schubert F, Köhler B. Ten lectures on impact-echo. J Nondestruct Eval 2008;27(1-3):5-21

[29] Piwakowski B, Safinowski P. Non-destructive non-contact air-coupled concrete evaluation by an ultrasound automated device. In: Proc 7th international symposium on non destructive testing in civil engineering. NDTCE09, Nantes; 2009. p. 603-8.

[30] Klauder JR, Price AC, Darlington S, Albersheim WJ. The theory and design of chirp radars. Bell Syst Tech J 1960;39:745-807.

[31] McMechan GA, Yedlin MJ. Analysis of dispersive waves by wave field transformation. Geophysics 1981;46:869-74.

[32] Mokhtar TA, Herrmann RB, Russel DR. Seismic velocity and q model for the shallow structure of the Arabian shield from short-period Rayleigh wave. Geophysics 1988;53(11):1379-87.

[33] Goueygou M, Soltani F, Lafhaj Z, Piwakowski B. Effect of geometrical diffraction on non contact measurements of ultrasonic attenuation in cement based materials. In: Proc NDTCE'9 non destructive testing in civil engineering, Nantes, France; 2009.

[34] Bodet L, Van Wijk K, Bitri A, Abraham O, Côte Ph, Grandjean G, et al. Surfacewave dispersion inversion limitations from laser-Doppler experiments. J Environ Eng Geophys 2005;10(2):151-62.

[35] Landau LD, Lifshitz EM. Theory of elasticity. New York: Pergamon; 1959.

[36] Kyung-Young Jhang. Nonlinear ultrasonic techniques for nondestructive assessment of micro damage in material. A review. Int J Precision Eng Manuf 2009;10(1):123-35.

[37] Kim J, Jacobs L, Qu J. Experimental characterization of fatigue damage in a nickel-base superalloy using nonlinear ultrasonic waves. J Acoust Soc Am 2006;120(3):1266-73.

[38] Scalerandi M, Gliozzi AS, Bruno CLE, Masera D, Bocca PG. A scaling method to enhance detection of a nonlinear elastic response. Appl Phys Lett 2008;92(10).

[39] Delsanto PP. Universality of nonclassic nonlinearity. Application to non destructive evaluations and ultrasonics. Springer; 2006.

[40] Van Den Abeele K, De Visscher J. Damage assessment in reinforced concrete using spectral and temporal nonlinear vibration techniques. Cem Concr Res 2000;30(9):1453-64.

[41] Payan C, Garnier V, Moysan J, Johnson PA. Applying nonlinear resonant ultrasound spectroscopy to improving thermal damage assessment in concrete. J Acoust Soc Am 2007;121(4):125-30.

[42] Wang G, Li SF. A penny-shapped cohesive cracks model for material damage. Theor Appl Fract Mech 2004;42(3):303-16.

[43] Payan C, Garnier V, Moysan J. Potential of nonlinear ultrasonic indicators for NonDestructive Testing of concrete. Adv Civil Eng 2010:238472.

[44] Van Den Abeele K, Sutin A, Carmeliet J, Johnson PA. Micro-damage diagnostics using nonlinear elastic wave spectroscopy (NEWS). NDT\&E Int 2001;34(4):239-48.

[45] Payan C, Garnier V, Moysan J. Effect of water saturation and porosity on the nonlinear elastic response of concrete. Cem Concr Res 2010;40(3):473-6.

[46] Breysse D, Larget M, Sbartai ZM, Lataste JF, Balayssac JP. Quality of NDT measurements and accuracy of concrete physical properties quantitative. Eur J Environ Civ En 2011;15(4):619-32.

[47] Garnier V, Chaix JF, Rossat M, Payan C, Corneloup G. Non destructive characterisation of concretes by ultrasonic wave's propagation. Mecanique Ind 2009;10:299-303.

[48] Checkroun M. Caracterisation mecaniques des premiers centimetres du beton avec des ondes de surface. These de doctorat de l'Ecole Centrale de Nantes 2008. 
[49] Chaix JF, Garnier V, Corneloup G. Ultrasonic wave propagation in heterogeneous solid media: theoretical analysis and experimental validation. Ultrasonics 2006;44(2):200-10.

[50] Philippidis TP, Aggelis DG. Experimental study of wave dispersion and attenuation in concrete. Ultrasonics 2005;43(7):584-95.
[51] Homand F, Duffaut P. Manuel de mécanique des roches. Tome 1: fondements. Comité français de mécanique des roches. Presses de l'Ecole des Mines de Paris; 2000.

[52] Murphy WM. Effects of partial water saturation on attenuation in Massilon sandstone and Vycor porous glass. J Acoust Soc Am 1982;71(6):1458-68. 